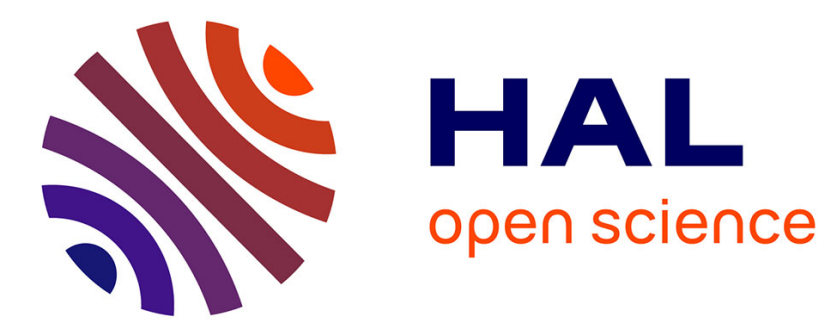

\title{
Haar negigeability of positive cones in Banach spaces
}

Jean Esterle, Etienne Matheron, P. Moreau

\section{To cite this version:}

Jean Esterle, Etienne Matheron, P. Moreau. Haar negigeability of positive cones in Banach spaces.

Algebra i Analiz, 2015, 27 (05), pp.32-68. hal-01067182

\section{HAL Id: hal-01067182 \\ https://hal.science/hal-01067182}

Submitted on 23 Sep 2014

HAL is a multi-disciplinary open access archive for the deposit and dissemination of scientific research documents, whether they are published or not. The documents may come from teaching and research institutions in France or abroad, or from public or private research centers.
L'archive ouverte pluridisciplinaire HAL, est destinée au dépôt et à la diffusion de documents scientifiques de niveau recherche, publiés ou non, émanant des établissements d'enseignement et de recherche français ou étrangers, des laboratoires publics ou privés. 


\title{
HAAR NEGLIGIBILITY OF POSITIVE CONES IN BANACH SPACES
}

\author{
JEAN ESTERLE, ÉTIENNE MATHERON, AND PIERRE MOREAU
}

\begin{abstract}
We discuss the Haar negligibility of the positive cone associated with a basic sequence in a separable Banach space. In particular, we show that up to equivalence, the canonical basis of $c_{0}$ is the only normalized subsymmetric unconditional basic sequence whose positive cone is not Haar null, and the only normalized unconditional basic sequence whose positive cone contains a translate of every compact set. We also show that an unconditional basic sequence with a non-Haar null positive cone has to be $c_{0}$-saturated in a very strong sense, and that every quotient of the space generated by such a sequence is $c_{0}$-saturated.
\end{abstract}

\section{INTRODUCTION}

The main topic of this paper is Haar negligibility, a quite well-known but still rather mysterious notion of smallness introduced by J. P. R. Christensen [Chr1] in the 1970's (and rediscovered much later in [HSY]). A Borel subset $A$ of a Polish abelian group $G$ is said to be Haar null if there exists a (Borel) probability measure $\mu$ on $G$ such that $\mu(A+x)=0$ for every $x \in G$. Any such measure $\mu$ is called a test measure for $A$.

If the group $G$ is locally compact, Haar negligibility turns out to be equivalent to negligibility with respect to the Haar measure of $G$. Haar null sets always have empty interior, and they form a $\sigma$-ideal, i.e. any countable union of Haar null sets is again Haar null. Also, a "Pettis' Lemma" is available: if a Borel set $A$ is not Haar null, then $A-A$ is a neighbourhood of 0 ; in particular, every compact subset of $G$ is Haar null if $G$ is not locally compact. Finally, it follows from the inner regularity of measures that if a Borel set $A \subset G$ contains a translate of every compact subset of $G$, then $A$ is not Haar null. (This is essentially the only known way of showing that a given set is not Haar null). We refer to [BeLi, Chapter 6] for more information.

In the present paper, we study Haar negligibility in the framework of separable Banach spaces.

Our work is motivated by beautiful results obtained by Matouskova and Stegall, which provide the following surprising link between the geometry of a Banach space and the Haar negligibility of its closed convex subsets with empty interior: A separable Banach space $X$ reflexive if and only if every closed convex subset of $X$ with empty interior is Haar null. More precisely: If $X$ is reflexive then every closed convex subset of $X$ with

2010 Mathematics Subject Classification. Primary 46B20, 46B09 Secondary 46B25, 46B45.

Key words and phrases. Haar negligibility, positive cone, Schauder base, Gauss negligibility. 
empty interior is Haar null ([M3]); and if $X$ is not reflexive, there exists a closed convex subset of $X$ with empty interior which contains a translate of every compact subset of $X$ $([\mathrm{MS}])$.

Thus, we see that as far as Haar negligibility is concerned, closed convex sets in reflexive Banach spaces are "uninteresting". However, this does not rule out the possibility that closed convex sets with empty interior taken from an interesting specific class turn out to be Haar null in some nonreflexive Banach spaces, and non-Haar null in some others.

With this in mind, a quite natural class of examples to consider is that of positive cones associated with basic sequences. If $\mathbf{e}=\left(e_{i}\right)_{i \geq 1}$ is a basic sequence in a Banach space $X$, the positive cone associated with $\mathbf{e}$ is the set of all $x \in X$ which can be written as $x=\sum_{1}^{\infty} x_{i} e_{i}$ with nonnegative coefficients $x_{i}$. This positive cone will be denoted by $Q^{+}(\mathbf{e})$. (That $Q^{+}(\mathbf{e})$ has indeed empty interior is easy to check).

In some sense, the real starting point of our study is the following well-known fact: the positive cone of $c_{0}$ is not Haar-null, whereas the positive cone of $\ell_{1}$ is Haar null. More generally, positive cones show-up naturally in the study of Haar negligibility, and play a crucial role in the proofs of the Matouskova and Stegall results. They are the main objects of study in the present paper.

Throughout the paper, we shall say that a positive cone $Q^{+}(\mathbf{e})$ in a Banach space $X$ is Haar null if it is Haar null in [e], the closed subspace of $X$ generated by e.

Ideally, one would like to characterize the basic sequences e whose positive cone is Haar null. (Note that since Haar negligibility is preserved under linear isomorphisms, this can be done only up to equivalence). However, this seems rather too ambitious, and we mostly concentrate on unconditional basic sequences.

Note that if $\mathbf{e}$ is unconditional then $Q^{+}(\mathbf{e})-Q^{+}(\mathbf{e})=[\mathbf{e}]$; so one would rather expect positive cones to be non-Haar null. Nevertheless, all our results suggest that the following "conjecture" might be true: up to equivalence, the only unconditional normalized basic sequence whose positive cone is not Haar null is the canonical basis of $c_{0}$.

We have been unable to prove this in full generality, but we believe that some of our results do support this conjecture rather convincingly. The most "important" ones seem to be the following.

- The conjecture holds true for subsymmetric unconditional basic sequences, and also for all unconditional basic sequences if "non-Haar negligibility" is replaced by the stronger property "to contain a translate of every compact set".

- A normalized unconditional basic sequence e whose positive cone is not Haar null has to be " $c_{0}$-saturated" in quite a strong sense: every normalized block-sequence of $\mathbf{e}$ has a subsequence equivalent to the canonical basis of $c_{0}$.

- If a Banach space $X$ has an unconditional basis whose positive cone is not Haar null, then every quotient of $X$ is $c_{0}$-saturated.

The paper is organized as follows. 
In Section 2, we introduce the notions and tools which will be used throughout the paper, and we recall some known results concerning the Haar negligibility of positive cones.

In Section 3 we give a characterization of those basic sequences whose positive cone contains a translate of every compact set, and we use it to show that the canonical basis of $c_{0}$ is (up to equivalence) the only normalized unconditional basic sequence with this property. We also observe that unconditionality cannot be dispensed with, one counterexample being the positive cone of the classical James space.

In Section 4 we obtain several results concerning unconditional basic sequences whose positive cone is not Haar null. We show in 4.1 that any block-sequence of such a sequence e also has a non-Haar null mositive cone, from which it follows that all block-sequences of e are both shrinking and non-boundedly complete. In 4.2 , we isolate a simple necessary condition for the non-Haar negligibility of a positive cone, which turns out to be crucial in our study. We use this condition to prove in a very elementary way that the positive cone of the Schreier space is Haar null, and that the canonical basis of $c_{0}$ is the only symmetric sequence with a non-Haar null positive cone. The $c_{0}$-saturation property stated above is established in 4.3, and the result concerning subsymmetric sequences follows immediately. In 4.4 , we prove a stronger $c_{0}$-saturation result. However, in 4.5 we give an example showing that this kind of $c_{0}$-saturation is not even sufficient to ensure the non-Haar negligibility of the positive cone.

In Section 5 we show that non-Haar negligibility of the positive cone associated with an unconditional basis entails $c_{0}$-saturation of the quotients.

Section 6 is independent of the rest of the paper. We give simple examples of Haar null positive cones admitting a Gaussian test measure. On the other hand, we observe that the positive cone associated to an arbitrary Schauder basis is never Gauss null.

Finally, we list a few natural questions in Section 7.

This work is based on Chapter 3 of the third author's $\mathrm{PhD}$ thesis [Mo].

\section{BACKGROUND}

2.1. Notations and terminology. We start by fixing some notations and recalling a few quite well-known notions from Banach space theory. Our references are [LiTz] and $[\mathrm{AK}]$.

Throughout the paper, the symbol $X$ will denote a Banach space with norm $\|\cdot\|$. As a rule, all Banach spaces are real, separable and infinite-dimensional.

For any family of vectors $\mathbf{e}=\left(e_{i}\right)_{i \in I}$ in $X$, we denote by $\left[e_{i} ; i \in I\right]$, or simply by $[\mathbf{e}]$, the closed linear span of $\left\{e_{i} ; i \in I\right\}$.

A sequence $\mathbf{e}=\left(e_{i}\right)_{i \geq 1} \subset X$ is a basic sequence if it is a Schauder basis of its closed linear span $[\mathbf{e}]$, i.e. every $x \in[\mathbf{e}]$ can be written in a unique way as $x=\sum_{i>1} x_{i} e_{i}$, where $x_{i} \in \mathbb{R}$ and the series is norm-convergent. If, further, every (convergent) series $\sum x_{i} e_{i}$ is unconditionnally convergent, the basic sequence $\mathbf{e}$ is said to be unconditional. A basic 
sequence $\left(e_{i}\right)$ is said to be normalized if $\left\|e_{i}\right\|=1$ for all $i$, and semi-normalized if it is bounded and $\inf _{i}\left\|e_{i}\right\|>0$.

If $\mathbf{e}=\left(e_{i}\right)$ is a basic sequence, we denote by $\left(e_{i}^{*}\right)$ the associated sequence of coordinate functionals on $[\mathbf{e}]$. Note that if $\inf _{i}\left\|e_{i}\right\|>0$ then $\left(e_{i}^{*}\right)$ is $w^{*}$ - null, i.e. $\left\langle e_{i}^{*}, x\right\rangle \rightarrow 0$ for every $x \in[\mathbf{e}]$.

The basis constant of $\mathbf{e}=\left(e_{i}\right)_{i \geq 1}$ is the number $K(\mathbf{e}):=\sup \left\{\left\|P_{n}\right\|, n \geq 1\right\}$, where $P_{n}$ is the canonical projection of $[\mathbf{e}]$ onto $\left[e_{i} ; 1 \leq i \leq n\right]$. This depends heavily on the given norm $\|\cdot\|$, but the formula $\|x\|:=\sup \left\{\left\|P_{n}(x)\right\|, n \geq 1\right\}$ defines an equivalent norm on $[\mathbf{e}]$ with respect to which the basis constant of $\mathbf{e}$ is equal to 1 . Since Haar negligibility is preserved under linear isomorphisms, it follows that for our purpose, we can safely restrict ourselves to basic sequences with basis constant equal to 1 .

Likewise, if $\mathbf{e}$ is unconditional, the unconditional basis constant of $\mathbf{e}$ is the number $K_{u}(\mathbf{e}):=\sup \left\{\left\|P_{I}\right\| ; I \subset \mathbb{N}\right\}$, where $P_{I}$ is the canonical projection of $[\mathbf{e}]$ onto $\left[e_{i} ; i \in I\right]$; and this can be made equal to 1 by replacing the original norm with the equivalent norm $\|x\|:=\sup \left\{\left\|P_{I}(x)\right\| ; I \subset \mathbb{N}\right\}$. When $K_{u}(\mathbf{e})=1$, the basic sequence $\mathbf{e}$ is said to be 1-unconditional.

If $\mathbf{e}$ is a basic sequence in $X$, we denote $\|\cdot\|^{\mathbf{e}}$ the restriction of $\|\cdot\|$ to the closed subspace $[\mathbf{e}] \subset X$. If $\mathbf{e}$ is normalized, we denote by $\|\cdot\|_{\infty}^{\mathbf{e}}$ the " $\ell_{\infty}$ norm built on $\mathbf{e} "$, i.e. the norm on $[\mathbf{e}]$ defined by

$$
\left\|\sum_{i \geq 1} x_{i} e_{i}\right\|_{\infty}^{\mathbf{e}}:=\sup _{i \geq 1}\left|x_{i}\right| .
$$

When $\mathbf{e}$ is a basis of $X$, we write $\|\cdot\|_{\infty}$ instead of $\|\cdot\|_{\infty}^{\mathbf{e}}$.

It is important to keep in mind that the original norm always dominates the $\ell_{\infty}$ norm built on a normalized basic sequence e; more precisely, we have

$$
\|\cdot\|_{\infty}^{\mathbf{e}} \leq 2 K(\mathbf{e})\|\cdot\|^{\mathbf{e}} .
$$

Two basic sequences $\left(e_{i}\right)_{i>1}$ and $\left(f_{i}\right)_{i \geq 1}$ (living on possibly different Banach spaces) are said to be equivalent if, for every sequence of scalars $\left(x_{i}\right)_{i \geq 1}$, the convergence of the series $\sum x_{i} e_{i}$ is equivalent to the convergence of the series $\sum x_{i} f_{i}$; in other words, if there is an isomorphism from $\left[e_{i} ; i \geq 1\right]$ onto $\left[f_{i} ; i \geq 1\right]$ sending $e_{i}$ to $f_{i}$. Note that the property "to have a Haar null positive cone" is invariant under equivalence.

If $\mathbf{e}=\left(e_{i}\right)_{i \geq 1}$ is a basic sequence, a block-sequence built on $\mathbf{e}$ is a sequence $\mathbf{f}=\left(f_{j}\right)_{j \geq 1}$ of the form

$$
f_{j}=\sum_{i=p_{j-1}}^{p_{j}-1} a_{i} e_{i},
$$

where $\left(p_{j}\right)_{j \geq 0}$ is an increasing sequence of integers and $\left(a_{i}\right)$ is a sequence of scalars. Any such sequence $\mathbf{f}$ is basic, with $K(\mathbf{f}) \leq K(\mathbf{e})$; and if $\mathbf{e}$ is unconditional then so is $\mathbf{f}$, with $K_{u}(\mathbf{f}) \leq K_{u}(\mathbf{e})$. 
Finally, recall that a basic sequence $\mathbf{e}=\left(e_{i}\right)$ is said to be shrinking if the linear span of $\left(e_{i}^{*}\right)$ is dense in the dual space of $[\mathbf{e}]$, and boundedly complete if the convergence of a series $\sum x_{i} e_{i}$ is equivalent to the boundedness of its partial sums.

By a famous result due to $\mathrm{R}$. C. James, a basic sequence generates a reflexive Banach space if and only if it is both shrinking and boundedly complete. Moreover, an unconditional basic sequence is non-shrinking if and only if it has a subsequence equivalent to the canonical basis of $\ell_{1}$, and non-boundedly complete if and only if it has a subsequence equivalent to the canonical basis of $c_{0}$.

2.2. Three useful facts. For the sake of future reference, we now recall three extremely useful results concerning basic sequences.

The first one is merely an observation:

Fact 2.1. If $\mathbf{e}=\left(e_{i}\right)_{i \geq 1}$ is an unconditional basic sequence, then every bounded sequence of scalars $\mathbf{a}=\left(a_{i}\right)_{i \geq 1}$ defines a "bounded multiplier" on $[\mathbf{e}]$; more precisely, for any convergent series $\sum x_{i} e_{i}$, the series $\sum a_{i} x_{i} e_{i}$ is convergent with

$$
\left\|\sum_{i \geq 1} a_{i} x_{i} e_{i}\right\| \leq K_{u}(\mathbf{e})\|\mathbf{a}\|_{\infty}\left\|\sum_{i \geq 1} x_{i} e_{i}\right\| .
$$

In particular, any fixed change of signs on the $e_{i}$ 's defines an isomorphism of $[\mathbf{e}]$. Since Haar negligibility is preserved under linear isomorphisms, it follows that the property "to have a Haar null positive cone" is invariant under changes of signs: if an unconditional basic sequence $\left(e_{i}\right)$ has a Haar null positive cone, then so does any sequence of the form $\left( \pm e_{i}\right)$.

The second result we want to state explicitely is the "principle of small perturbations", which is a standard tool for establishing the equivalence of two basic sequences:

Lemma 2.2. Let $\mathbf{e}=\left(e_{i}\right)_{i \geq 1}$ be a normalized basic sequence in $X$. If $\mathbf{f}=\left(f_{i}\right)_{i \geq 1}$ is a sequence in $X$ such that $\eta:=\sum_{1}^{\infty}\left\|e_{i}-f_{i}\right\|<\frac{1}{2 K(\mathbf{e})}$, then $\mathbf{f}$ is basic and equivalent to $\mathbf{e}$. Moreover, for every convergent series $\sum x_{i} f_{i}$, one has the following estimate:

$$
(1-2 K(\mathbf{e}) \eta)\left\|\sum_{i=1}^{\infty} x_{i} e_{i}\right\| \leq\left\|\sum_{i=1}^{\infty} x_{i} f_{i}\right\| \leq 2 K(\mathbf{e})\left\|\sum_{i=1}^{\infty} x_{i} e_{i}\right\| .
$$

The above estimate is not explicitely stated in $[\mathrm{AK}]$ or $[\mathrm{LiTz}]$, but it follows easily from the proofs of the principle of small perturbations given therein.

Finally, let us recall the so-called "Bessaga-Pelczynski selection principle":

Lemma 2.3. If $\mathbf{e}$ is a basic sequence, then any normalized sequence $\left(y_{n}\right) \subset[\mathbf{e}]$ such that $\left\langle e_{i}^{*}, y_{n}\right\rangle \underset{n \rightarrow \infty}{\longrightarrow} 0$ for all $i \in \mathbb{N}$ admits a basic subsequence equivalent to a (normalized) block-sequence of $\mathbf{e}$.

This is often stated with a weakly null sequence $\left(y_{n}\right)$, but the greater generality is useful; see $[\mathrm{AK}]$. 
2.3. Some Haar null and non-Haar null positive cones. To conclude this background section, we now state some known results concerning the Haar negligibility of positive cones.

Proposition 2.4. Let $\mathbf{e}=\left(e_{i}\right)_{i>1}$ be a normalized basic sequence.

(1) If $\mathbf{e}$ is equivalent to the canonical basis of $c_{0}$, then $Q^{+}(\mathbf{e})$ contains a translate of every compact subset of $[\mathbf{e}]$, and so $Q^{+}(\mathbf{e})$ is not Haar null.

(2) If $\mathbf{e}$ is equivalent to the canonical basis of $\ell_{p}, 1 \leq p<\infty$, then $Q^{+}(\mathbf{e})$ is Haar null. More generally, $Q^{+}(\mathbf{e})$ is Haar null as soon as e dominates the canonical basis of $\ell_{p}$, i.e. there exists a finite constant $M$ such that

$$
\sum_{i}\left|x_{i}\right|^{p} \leq M\left\|\sum_{i} x_{i} e_{i}\right\|^{p}
$$

for every finite sequence of scalars $\left(x_{i}\right)$.

(3) If $[\mathbf{e}]$ is reflexive, then $Q^{+}(\mathbf{e})$ is Haar null.

(4) If $\mathbf{e}$ is unconditional and satisfies

$$
\lim _{k \rightarrow \infty} \inf _{n_{1}<\cdots<n_{k}}\left\|\sum_{i=1}^{k} e_{n_{i}}\right\|=\infty,
$$

then $Q^{+}(\mathbf{e})$ is Haar null.

Part (1) is almost obvious; see [BeLi, p. 132]. Part (2) can be found in [BorN] and [M1] (see also [BeLi]). Part (3) follows from the main result of [M3], and (4) can be found in [M2].

Concerning (1), will show in Section 3 that the canonical basis of $c_{0}$ is in fact the only unconditional basic sequence for which the positive cone contains a translate of every compact set. We will also see that (2), (3) and (4) can be obtained as applications of Proposition 4.6 and Theorem 4.9 below. (However, the direct proof of (3) given in [BeLi, Prop. 6.8] provides an explicit test measure for the positive cone).

\section{Positive cones containing a translate of EVERy Compact Set}

In this section, we study the positive cones $Q^{+}(\mathbf{e})$ containing a translate of every compact set. By this, we mean of course that $Q^{+}(\mathbf{e})$ contains a translate of every compact subset of $[\mathbf{e}]$. As already mentioned, this is a strong way of being non-Haar null.

The main result of this section is the following.

Theorem 3.1. Let $\mathbf{e}=\left(e_{i}\right)_{i \geq 1}$ be a normalized basic sequence. The positive cone $Q^{+}(\mathbf{e})$ contains a translate of every compact set if and only if there exists a sequence of real numbers $\left(\lambda_{i}\right)_{i \geq 1}$, with $\lambda_{i} \geq 1$ for all $i$, such that

$$
\sup _{n \geq 1}\left\|\sum_{i=1}^{n} \lambda_{i} e_{i}\right\|<\infty .
$$


From this, it is easy to deduce

Corollary 3.2. Up to equivalence, the only normalized unconditional basic sequence whose positive cone contains a translate of every compact set is the canonical basis of $c_{0}$.

Proof. Let e be a normalized unconditional basic sequence such that $Q^{+}(\mathbf{e})$ contains a translate of every compact set, and set $X:=[\mathbf{e}]$. Without loss of generality, we may assume that $\mathbf{e}$ is 1-unconditional.

We already know that the original norm $\|\cdot\|$ of $X$ dominates the norm $\|\cdot\|_{\infty}$, so we just have to show that $\|\cdot\|_{\infty}$ also dominates $\|\cdot\|$. Let $\left(\lambda_{i}\right)_{i \geq 1}$ be the sequence given by Theorem 3.1, and set

$$
M:=\sup _{n \geq 1}\left\|\sum_{i=1}^{n} \lambda_{i} e_{i}\right\|<\infty .
$$

For every $x=\sum_{i \geq 1} x_{i} e_{i} \in X \backslash\{0\}$ and all $n \geq 1$, we have

$$
P_{n}(x):=\sum_{i=1}^{n} x_{i} e_{i}=\sum_{i=1}^{n} \frac{x_{i}}{\lambda_{i}} \lambda_{i} e_{i} .
$$

If we set $a_{i}:=x_{i} / \lambda_{i}$, then $\left|a_{i}\right| \leq\|x\|_{\infty}$ and so, by Fact 2.1:

$$
\left\|P_{n}(x)\right\| \leq\|x\|_{\infty}\left\|\sum_{i=1}^{n} \lambda_{i} e_{i}\right\| \leq M\|x\|_{\infty} .
$$

Hence, we obtain $\|x\|=\lim _{n \rightarrow \infty}\left\|P_{n}(x)\right\| \leq M\|x\|_{\infty}$.

The proof of Theorem 3.1 relies on the following lemma. Here and afterwards, for any positive number $r$, we set

$$
Q_{r}^{+}(\mathbf{e}):=Q^{+}(\mathbf{e}) \cap \bar{B}(0, r) .
$$

Lemma 3.3. Let $\mathbf{e}$ be a Schauder basis of a Banach space $X$, and let $D$ be any dense subset of $\bar{B}(0,1)$. The following are equivalent.

(i) The cone $Q^{+}(\mathbf{e})$ contains a translate of every compact set.

(ii) There exists $R>0$ such that $Q_{R}^{+}(\mathbf{e})$ contains a translate of every compact subset of $\bar{B}(0,1)$.

(iii) There exists $M>0$ such that $Q_{M}^{+}(\mathbf{e})$ contains a translate of every finite subset of $\bar{B}(0,1) \cap D$.

Moreover if condition (iii) is satisfied, then (ii) holds with $R=2 M$.

The proof of this lemma is quite nontrivial; but it is essentially the same as that of [MS, Theorem 3] and is therefore omitted.

Proof of Theorem 3.1. Without loss of generality, we may assume that the basis constant of $\mathbf{e}$ is equal to 1. 
Assume first that $Q^{+}(\mathbf{e})$ contains a translate of every compact subset of $X:=[\mathbf{e}]$. By Lemma 3.3, there exists $M>0$ such that $Q_{M}^{+}$contains a translate of every finite subset of $D:=\bar{B}(0,1) \cap \operatorname{span}\left(e_{i} ; i \geq 1\right)$.

For every $k \geq 1$, set $F_{k}:=\left\{-e_{i} ; 1 \leq i \leq k\right\}$. This is a finite subset of $D$, so there exists $z_{k}=\sum_{i=1}^{\infty} z_{i, k} e_{i} \in X$ such that $z_{k}+F_{k} \subset Q_{M}^{+}(\mathbf{e})$. Then $\left\|z_{k}\right\| \leq M+1$, and so $\left|z_{i, k}\right| \leq 2(M+1)$ since $K(\mathbf{e})=1$. Moreover, since $z_{k}+F_{k} \subset Q^{+}(\mathbf{e})$ we have $z_{i, k} \geq 1$ whenever $k \geq i$; hence $1 \leq z_{i, k} \leq 2(M+1)$ for $i \geq 1$ and $k \geq i$.

By a diagonal process, one can find an increasing sequence of integers $\left(p_{k}\right)_{k \geq 1}$ such that for every $i \geq 1$, the sequence $\left(z_{i, p_{k}}\right)_{k \geq 1}$ converges to some real number $\lambda_{i} \geq 1$ as $k \rightarrow \infty$. Let us show that the sequence $\left(\lambda_{i}\right)_{i \geq 1}$ has the required property, i.e. that the partial sums of the series $\sum \lambda_{i} e_{i}$ are uniformly bounded.

For any $n, k \geq 1$, we have

$$
\begin{aligned}
\left\|\sum_{i=1}^{n} \lambda_{i} e_{i}\right\| & \leq\left\|\sum_{i=1}^{n}\left(\lambda_{i}-z_{i, p_{n}}\right) e_{i}\right\|+\left\|P_{n}\left(z_{p_{k}}\right)\right\| \\
& \leq n \sup \left\{\left|\left(\lambda_{i}-z_{i, p_{k}}\right)\right| ; 1 \leq i \leq n\right\}+\left\|z_{p_{k}}\right\| \\
& \leq n \sup \left\{\left|\left(\lambda_{i}-z_{i, p_{k}}\right)\right| ; 1 \leq i \leq n\right\}+M+1 .
\end{aligned}
$$

Since this inequality holds for every $k \geq 1$, we obtain

$$
\left\|\sum_{i=1}^{n} \lambda_{i} e_{i}\right\| \leq M+1 \quad \text { for all } n \geq 1 .
$$

We now prove the converse. Suppose that there exists $M>0$ and a sequence of real numbers $\left(\lambda_{i}\right)_{i \geq 1}$ with $\lambda_{i} \geq 1$, such that $\left\|\sum_{i=1}^{n} \lambda_{i} e_{i}\right\| \leq M$ for every $n \geq 1$.

Set again $D:=\bar{B}(0,1) \cap \operatorname{span}\left(e_{i} ; i \geq 1\right)$. Then $R:=1+2 M$ satisfies property (iii) of Lemma 3.3. Indeed let $F$ be any finite subset of $D$. One can find $m \geq 1$ such that $\left\langle e_{n}^{*}, x\right\rangle=0$ for every $x \in F$ if $n \geq m$. Since $\left|\left\langle e_{n}^{*}, x\right\rangle\right| \leq 2$ for all $n \geq 1$, if $x \in F$ (because $\|x\| \leq 1)$, we see that $F+2 \sum_{i=1}^{m} \lambda_{i} e_{i} \subset Q_{1+2 M}^{+}(\mathbf{e})$.

We conclude this section by a simple example showing that Corollary 3.2 breaks down if we drop the unconditionality assumption.

Example 3.4. The positive cone associated to the canonical basis of the James space $\mathcal{J}$ contains a translate of every compact set.

Proof. Recall that $\mathcal{J}$ is the space of all sequences of real numbers $x=\left(x_{i}\right)_{i \geq 1}$ which converge to 0 and satisfy the following condition:

$$
\|x\|_{\mathcal{J}}:=\sup _{m \in \mathbb{N}} \sup \left\{\left[\left(x_{p_{2}}-x_{p_{1}}\right)^{2}+\cdots+\left(x_{p_{m}}-x_{p_{m-1}}\right)^{2}\right]^{1 / 2} ; 1 \leq p_{1} \cdots<p_{m}\right\}<\infty .
$$


We refer to e.g. [LiTz, p. 25] for more details on the James space.

The canonical basis $\mathbf{e}=\left(e_{i}\right)_{i \geq 0}$ is a normalized Schauder basis of $\mathcal{J}$, and for every $n \geq 1$ we have

$$
\left\|\sum_{i=1}^{n} e_{i}\right\|_{\mathcal{J}}=1 .
$$

It follows then from Theorem 3.1 that $Q^{+}(\mathbf{e})$ contains a translate of every compact set.

\section{Positive Cones Which are not HaAR NUlL}

We now address the question of characterizing the basic sequences whose positive cone is not Haar null. We obtain a complete answer in the case of subsymmetric unconditional sequences: within this class of sequences, the canonical basis of $c_{0}$ is the only one whose positive cone is not Haar null. We also show that an unconditional basic sequence whose positive cone is not Haar null has to be "very close" to the canonical basis of $c_{0}$, in a sense that will be made precise.

4.1. Haar negligibility and block-sequences. The following result shows that for unconditional basic sequences, Haar negligibility of the positive cone can be detected by looking at any block-sequence.

Proposition 4.1. Let $\mathbf{e}=\left(e_{i}\right)_{i \geq 1}$ be an unconditional basic sequence. If $\mathbf{e}$ admits a block-sequence $\mathbf{f}$ whose positive cone is Haar null, then $Q^{+}(\mathbf{e})$ is Haar null.

Proof. Set $X:=[\mathbf{e}]$, and let $Y$ be the closed subspace of $X$ generated by the blocksequence $\mathbf{f}=\left(f_{j}\right)_{j \geq 1}$. There exists an increasing sequence of integers $\left(p_{j}\right)_{j \geq 0}$, with $p_{0}=1$, such that $f_{j}=\sum_{i=p_{j-1}}^{p_{j}-1} a_{i} e_{i}$; and since Haar negligibility is invariant under any change of signs on the $e_{i}$ 's, we may assume that $a_{i} \geq 0$ for all $i$.

By assumption, one can find a probability measure $\mu$ on $Y$ such that $\mu\left(Q^{+}(\mathbf{f})+y\right)=0$ for every $y \in Y$. Denote by $\nu$ the measure $\mu$ viewed as a probability measure on $X$; that is, $\nu(A)=\mu(A \cap Y)$ for every Borel set $A \subset X$. We show that $\nu$ is a test measure for $Q^{+}(\mathbf{e})$.

Since $\mathbf{e}$ is unconditional, we can write any $x \in X$ as $x=x^{+}+x^{-}$, where $x^{+} \in Q^{+}(\mathbf{e})$ and $x^{-} \in Q^{-}(\mathbf{e})$. Then $x+Q^{+}(\mathbf{e}) \subset x^{-}+Q^{+}(\mathbf{e})$, and so

$$
\nu\left(x+Q^{+}\right) \leq \nu\left(x^{-}+Q^{+}\right) .
$$

Hence, it is enough to check that $\nu\left(x+Q^{+}(\mathbf{e})\right)=0$ for every $x \in Q^{-}(\mathbf{e})$. This follows immediately from the following

Fact. Given $x \in Q^{-}(\mathbf{e})$, one can find $w \in X$ such that $\left(x+Q^{+}(\mathbf{e})\right) \cap Y \subset w+Q^{+}(\mathbf{f})$.

Indeed, once this is known one just has to write

$$
\nu\left(x+Q^{+}(\mathbf{e})\right)=\mu\left(\left(x+Q^{+}(\mathbf{e})\right) \cap Y\right) \leq \mu\left(w+Q^{+}(\mathbf{f})\right)=0 .
$$


Proof of Fact. Fix $x \in Q^{-}(\mathbf{e})$, and let $z \in\left(x+Q^{+}(\mathbf{e})\right) \cap Y$ be arbitrary. Write

$$
z=\sum_{i=1}^{\infty}\left(x_{i}+\lambda_{i}\right) e_{i}=\sum_{j=1}^{\infty} z_{j} f_{j}=\sum_{j=1}^{\infty} z_{j}\left(\sum_{i=p_{j-1}}^{p_{j}-1} a_{i} e_{i}\right)
$$

where $\lambda_{i} \geq 0$ for all $i$. Then $z_{j} a_{i}-x_{i}=\lambda_{i} \geq 0$ for every $j \geq 1$ and all $i \in\left[p_{j-1}, p_{j}\right)$. So we get for $j \geq 1$,

$$
w_{j}:=\sup \left\{\frac{x_{i}}{a_{i}} ; p_{j-1} \leq i<p_{j}, a_{i} \neq 0\right\} \leq z_{j}
$$

Since $x_{i} \leq 0$ for all $i$, the series $\sum\left|x_{i}\right| e_{i}$ is convergent and $\sum_{i=1}^{\infty}\left|x_{i}\right| e_{i}=-x$. It follows from Fact 2.1 that the series $\sum_{j \geq 1}\left(\sum_{i=p_{j-1}}^{p_{j}-1}\left|w_{j}\right| a_{i} e_{i}\right)=\sum\left|w_{j}\right| y_{j}$ is convergent, and so the series $\sum w_{j} y_{j}$ is also convergent in $Y$. Set $w:=\sum_{j=1}^{\infty} w_{j} y_{j}$. Note that $w$ depends only on $x$ and $\mathbf{f}$ (not on the point $z$ we are considering).

Using (4.1), we see that $z=w+\sum_{j=1}^{\infty}\left(z_{j}-w_{j}\right) y_{j} \in w+Q^{+}(\mathbf{f})$, which concludes the proof.

We know that the positive cone of $\ell_{1}$ is Haar null, and that positive cones in reflexive spaces are also Haar null. By James' characterization of reflexivity, we deduce

Corollary 4.2. If $\mathbf{e}$ is an unconditional basic sequence such that $Q^{+}(\mathbf{e})$ is not Haar null, then every block-sequence of $\mathbf{e}$ is both shrinking and non-boundedly complete.

Since an unconditional basic sequence is non-boundedly complete if and only if it has a block-sequence equivalent to the canonical basis of $c_{0}$, it follows that if $\mathbf{e}$ is unconditional and $Q^{+}(\mathbf{e})$ is not Haar null, then every block-sequence of e admits a block-sequence equivalent to the canonical basis of $c_{0}$. We will see in Section 4.4 that $\mathbf{e}$ has in fact a much stronger " $c_{0}$-saturation" property.

4.2. The $(\delta, R)$-condition. Matouskova observed in [M2] that a Borel subset $A$ of a Polish abelian group $G$ is Haar null if and only if the following holds: for every $\delta>0$ and every $r>0$, there exists a probability measure $\mu$ supported by the open ball $B(0, r)$ such that $\mu(A+g) \leq \delta$ for every $g \in G$. From this, it is easy to deduce

Lemma 4.3. Let $\mathbf{e}$ be a normalized basic sequence. The cone $Q^{+}(\mathbf{e})$ is not Haar null if and only if there exist $\delta>0$ and $R>0$ satisfying the following property:

(*) For every probability measure $\mu$ on $X$ supported on $\bar{B}(0,1)$, there exists $x \in X$ such that $\mu\left(x+Q_{R}^{+}(\mathbf{e})\right) \geq \delta$.

In this situation we have $\|x\| \leq 1+R$. 
Proof. Since a countable union of Haar null sets is Haar null, $Q^{+}(\mathbf{e})$ is not Haar null if and only if $Q_{n}^{+}(\mathbf{e})$ is not Haar null for some integer $n \geq 1$.

If $Q_{n}^{+}(\mathbf{e})$ is not Haar null, it follows from Matouskova's result mentioned above that there exist $\delta>0$ and $r>0$ such that for every probability mesure $\mu$ on $X$ of support contained in $\bar{B}(0, r)$, there exists $x \in X$ such that $\mu\left(x+Q_{n}^{+}\right) \geq \delta$. This gives the required condition with $R:=n / r$. Conversely if $(*)$ holds then Matouskova's result implies that $Q_{R}^{+}(\mathbf{e})$ is not Haar null, and so $Q^{+}(\mathbf{e})$ is not Haar null either.

The inequality $\|x\| \leq 1+R$ follows from the triangle inequality.

In what follows we will consider a weakened version of condition $(*)$ above, where we only allow a special type of measures.

Definition 4.4. Let $\delta>0$ and $R>0$. We say that a normalized basic sequence $\mathbf{e}=\left(e_{i}\right)_{i \geq 1}$ satisfies the $(\delta, R)$-condition if for every probability measure $\mu$ having finite support contained in $\left\{-e_{i} ; i \geq 1\right\}$, there exists $x \in X$ (necessarily satisfying $\|x\| \leq 1+R$ ) such that $\mu\left(x+Q_{R}^{+}\right) \geq \delta$.

The following observation shows the relevance of this condition to our matters.

Observation 4.5. Let $\mathbf{e}=\left(e_{i}\right)$ be a normalized basic sequence.

(i) If $Q^{+}(\mathbf{e})$ is not Haar null, then e satisfies the $(\delta, R)$-condition for some $\delta, R>0$.

(ii) If $\mathbf{e}$ is unconditional and if $Q^{+}(\mathbf{e})$ is not Haar null, there exist $\delta, R>0$ such that all normalized block-sequences of e satisfy the $(\delta, R)$-condition.

Proof. Part (i) follows immediately from Lemma 4.3. To prove (ii), argue by contradiction and use Proposition 4.1.

When e is unconditional, this implies a condition on the norm of all finite sums of the form $\sum_{i \in J} e_{i}$.

Proposition 4.6. Let $\mathbf{e}=\left(e_{i}\right)$ be a normalized, 1-unconditional basic sequence, and assume that e satisfies the $(\delta, R)$-condition for some $\delta, R>0$. Then the following facts hold.

(a) For every finite set $I \subset \mathbb{N}$, one can find $J \subset I$ such that

$$
|J| \geq \delta|I| \quad \text { and } \quad\left\|\sum_{i \in J} e_{i}\right\| \leq 1+R .
$$

(b) If $\delta=1$, then $\mathbf{e}$ is equivalent to the canonical basis of $c_{0}$.

(c) If $\delta<1$ then, for any finite set $I \subset \mathbb{N}$, one has the estimate

$$
\left\|\sum_{i \in I} e_{i}\right\| \leq \frac{1+R}{-\log (1-\delta)} \log (|I|)+2(1+R) .
$$


Proof. (a) Set $\lambda_{i}=\frac{1}{|I|}$ for $i \in I$. Then $\mu=\sum_{i \in I} \lambda_{i} \delta_{-e_{i}}$ is a probability measure with finite support contained in $\left\{-e_{i}, i \geq 1\right\}$. So there exists $x \in X$ such that $\mu\left(x+Q_{R}^{+}\right) \geq \delta$. This implies that there exists $J \subset I$ such that $|J| \geq \delta|I|$ and such that $-e_{j} \in x+Q_{R}^{+}$for all $j \in J$. So $\left\langle e_{j}^{*}, x\right\rangle \leq-1$ for all $j \in J$ (and hence $\left|\left\langle e_{j}^{*}, x\right\rangle\right| \geq 1$ ). Since $\mathbf{e}$ is 1-unconditional and since $\|x\| \leq 1+R$, it follows from Fact 2.1 that $\left\|\sum_{i \in J} e_{i}\right\| \leq 1+R$.

(b) If $\delta=1$, then for every finite set $I$ we have $\left\|\sum_{i \in I} e_{i}\right\| \leq 1+R$. By 1-unconditionality, this implies that $\|\cdot\| \leq(1+R)\|\cdot\|_{\infty}$, and hence $\mathbf{e}$ is equivalent to the canonical basis of $c_{0}$.

(c) If $\delta<1$, we construct a family of disjoint subsets of $I$ in the following way: set $I_{0}:=I$ and choose $J_{0} \subset I_{0}$ according to (i). Then set $I_{1}=I_{0}-J_{0}$ and we choose $J_{1} \subset I_{1}$ in the same way, and so on. At step $k$, we thus have $\left|I_{k}\right| \leq(1-\delta)\left|I_{k-1}\right|,\left|I_{k}\right| \leq(1-\delta)^{k}|I|$ and $\left\|\sum_{i \in J_{k}} e_{i}\right\| \leq 1+R$

We stop when $I_{k+1}$ is empty, so at the latest when $(1-\delta)^{k}|I| \leq 1$, which gives $k \geq \frac{\log (|I|)}{-\log (1-\delta)}$. So we can choose $k \leq \frac{\log (|I|)}{-\log (1-\delta)}+1$. This gives

$$
\left\|\sum_{i \in I} e_{i}\right\| \leq \sum_{j=0}^{k}\left\|\sum_{i \in J_{j}} e_{i}\right\| \leq(k+1)(1+R) \leq \frac{1+R}{-\log (1-\delta)} \log (|I|)+2(1+R) .
$$

Remark 1. Part (4) of Proposition 2.4 follows immediately from (a).

Remark 2. If $\mathbf{e}=\left(e_{i}\right)$ satisfies the assumption of Proposition 4.6, then (c) shows that

$$
\lim _{|I| \rightarrow \infty}\left\|\frac{1}{|I|} \sum_{i \in I} e_{i}\right\|=0 .
$$

Sequences e satisfying this property are called Blum-Hanson sequences in [LMP]. They are quite interesting objects of study.

Remark 3. For every normalized basic sequence $\mathbf{e}=\left(e_{i}\right)$ and any $M \in \mathbb{R}^{+}$, let us denote by $\mathcal{I}_{M}(\mathbf{e})$ the family of all subsets $\sigma$ of $\mathbb{N}$ such that

$$
\left\|\sum_{i \in I} e_{i}\right\| \leq M \quad \text { for every finite set } I \subset \sigma .
$$

Note that if $\mathbf{e}$ is unconditional and if $\sigma \in \mathcal{I}_{M}(\mathbf{e})$ is infinite, then $\left(e_{i}\right)_{i \in \sigma}$ is equivalent to the canonical basis of $c_{0}$. So our "main conjecture" would be proved if one could show the following: if $\mathbf{e}$ is a normalized basic sequence such that $Q^{+}(\mathbf{e})$ is not Haar null, then one can partition $\mathbb{N}$ into finitely many sets from $\bigcup_{M>0} \mathcal{I}_{M}(\mathbf{e})$. Even though this is of course very unlikely to be relevant, it is hard not to notice the formal analogy with the 
"paving" formulation of the famous and recently solved Kadison-Singer Problem. Note that, by standard arguments, it would be enough to show that any ultrafilter $\mathcal{U}$ on $\mathbb{N}$ contains a set from $\bigcup_{M>0} \mathcal{I}_{M}(\mathbf{e})$.

Assuming that e is unconditional and that $Q^{+}(\mathbf{e})$ is not Haar null (so that e satisfies some $(\delta, R)$-condition), Proposition 4.6 (a) shows that there exists a constant $M$ such that $\mathcal{I}_{M}(\mathbf{e})$ has an interesting "largeness" property, namely that inside any finite set $I \subset \mathbb{N}$, one can find a set $J \in \mathcal{I}_{M}(\mathbf{e})$ with comparable cardinality; but this is definitely not enough to get a partition result.

In the same spirit (and under the same assumptions on e), the argument of $[\mathrm{BouTz}$, Corollary 1.4] shows the following: there exists a constant $M$, a probability measure $\mathfrak{m}$ on the compact set $\mathcal{I}_{M}(\mathbf{e}) \subset 2^{\mathbb{N}}$ and a constant $\alpha>0$ such that

$$
\mathfrak{m}\left(\left\{\sigma \in \mathcal{I}_{M}(\mathbf{e}) ; i \in \sigma\right\}\right) \geq \alpha \quad \text { for every } i \in \mathbb{N} .
$$

This is another "largeness" property of the family $\mathcal{I}_{M}(\mathbf{e})$, that makes perhaps plausible the existence of a suitable partition.

We now show that Proposition 4.6 can be used to characterize the symmetric basic sequences whose positive cone is Haar null. Recall that a basic sequence $\left(e_{i}\right)$ is said to be symmetric if, for any permutation $\pi$ of the integers, the sequence $\left(e_{\pi(i)}\right)$ is equivalent to $\left(e_{i}\right)$. In this case (see [LiTz, Chapter 3]), there exists a constant $C>0$ such that for every convergent series $\sum_{i \geq 1} x_{i} e_{i}$ and every permutation $\pi: \mathbb{N} \rightarrow \mathbb{N}$ we have

$$
\frac{1}{C}\left\|\sum_{i \geq 1} x_{i} e_{\pi(i)}\right\| \leq\left\|\sum_{i \geq 1} x_{i} e_{i}\right\| \leq C\left\|\sum_{i \geq 1} x_{i} e_{\pi(i)}\right\| .
$$

Every symmetric sequence is unconditional, but not conversely (consider any enumeration of the "canonical" basis of $l_{1} \oplus c_{0}$ ).

Corollary 4.7. Up to equivalence, the only normalized and symmetric basic sequence whose positive cone is not Haar null is the canonical basis of $c_{0}$.

Proof. Let $\mathbf{e}=\left(e_{i}\right)$ be a normalized, symmetric sequence such that $Q^{+}(\mathbf{e})$ is not Haar null. Assume without loss of generality that $\mathbf{e}$ is 1-unconditional. Choose $\delta, R>0$ such that $\mathbf{e}$ satisfies the $(\delta, R)$-condition. By proposition 4.6 and since $K_{u}(\mathbf{e})=1$, one can find for every integer $p \geq 1$ a finite set $I_{p} \subset \mathbb{N}$ of cardinality $p$ such that $\left\|\sum_{i \in I_{p}} e_{i}\right\| \leq 1+R$. Since e symmetric, there exists a finite constant $C$ such that, for every finite set $I$ of integers of cardinality $p$, we have

$$
\left\|\sum_{i \in I} e_{i}\right\| \leq C\left\|\sum_{i \in I_{p}} e_{i}\right\|
$$


So the finite sums $\sum_{i \in I} e_{i}$ are uniformly bounded, which implies (by unconditionality) that the given norm $\|\cdot\|^{\mathbf{e}}$ on $[\mathbf{e}]$ is dominated by the norm $\|\cdot\|_{\infty}^{\mathbf{e}}$. Hence these two norms are equivalent, and $\mathbf{e}$ is equivalent to the canonical basis of $c_{0}$.

As another of illustration of Proposition 4.6, we now show that the positive cone associated with the canonical basis of the Schreier space $\mathcal{S}$ is Haar null.

Let us first recall the definition of $\mathcal{S}$. Let $c_{00}$ be the linear space of all real sequences with finite support. For $x=\left(x_{i}\right)_{i \geq 1} \in c_{00}$, set

$$
\|x\|_{\mathcal{S}}:=\sup \left\{\sum_{i=1}^{p}\left|x_{k_{i}}\right|: p \geq 1, p \leq k_{1}<\ldots<k_{p}\right\} .
$$

Then $\|\cdot\|_{\mathcal{S}}$ is a norm on $c_{00}$, and the Schreier space $\mathcal{S}$ is the completion of $c_{00}$ with respect to this norm. The canonical basis $\left(e_{i}\right)$ of $c_{00}$ is a normalized, 1-unconditional basis of $\mathcal{S}$, and it is also shrinking.

Corollary 4.8. The positive cone of the Schreier space is Haar null.

Proof. Let $n \geq 1$, and let $I \subset \mathbb{N}$ be a set of cardinality $2 n$. Then the set $I \cap[n, \infty)$ contains at least $n$ elements of $I$. It follows from the definition of $\|\cdot\|_{\mathcal{S}}$ that we have:

$$
\left\|\sum_{i \in I} e_{i}\right\|_{\mathcal{S}} \geq n
$$

Since $n$ is arbitrary, the result now follows from Proposition 4.6.

4.3. Extracting $c_{0}$-subsequences. In this section, we show that a normalized unconditional basic sequence whose positive cone is not Haar null has a wealth of subsequences equivalent to the canonical basis of $c_{0}$. This will follow easily from the following result.

Theorem 4.9. Let $\mathbf{e}$ be a normalized basic sequence, and assume that $\mathbf{e}$ satisfies the $(\delta, R)$-condition for some $\delta, R>0$. Then there exists an increasing sequence of integers $\left(m_{j}\right)_{i \geq 1}$ and a sequence $\left(x_{n}\right)_{n \geq 1} \subset \bar{B}(0,1+R)$ such that

$$
\left\langle e_{m_{j}}^{*}, x_{n}\right\rangle \geq 1 \text { for every } n \geq 1 \text { and all } j \in\{1, \ldots, n\} \text {. }
$$

Remark. It follows immediately from this result that a normalized Schauder basis of a reflexive Banach space cannot satisfy a $(\delta, R)$-condition, which gives part (3) of Proposition 2.4. Indeed let $X$ be a Banach with a normalized Schauder basis $\mathbf{e}=\left(e_{i}\right)_{i \geq 1}$, and assume that e satisfies the $(\delta, R)$-condition for some $\delta, R>0$. If the space $X$ were reflexive, it would be possible to extract from the sequence $\left(x_{n}\right)_{n \geq 1}$ given by theorem 4.9 a subsequence converging weakly to some $x \in X$. Then we would have $\left\langle e_{m_{j}}^{*}, x\right\rangle \geq 1$ for every $j \geq 1$, a contradiction since $\left\langle e_{m_{j}}^{*}, x\right\rangle \rightarrow 0$ (recall that $\mathbf{e}$ is normalized).

Part (2) of Proposition 2.4 is also a consequence of Theorem 4.9. Indeed, let $\mathbf{e}=\left(e_{i}\right)$ be a normalized basic sequence which dominates the canonical basis of $\ell_{p}$. If e were to satisfy some $(\delta, R)$-condition, then the sequence $\left(x_{n}\right)_{n \geq 1}$ given by 4.9 would satisfy 
$\sum_{i \geq 1}\left|\left\langle e_{i}^{*}, x_{n}\right\rangle\right|^{p} \geq n$ for every $n \geq 1$, which is impossible because $\left(x_{n}\right)$ is bounded. Hence e does not satisfy any $(\delta, R)$-condition, so $Q^{+}(\mathbf{e})$ is Haar null.

Theorem 4.9 is in fact a simple consequence of a classical combinatorial lemma due to V. Ptak, which was already an essential tool in [M3].

Lemma 4.10. Let $W$ be an infinite set, and let $\mathfrak{V}$ be a family of subsets of $W$. Denote by $\mathbf{P}(W)$ be the set of all finitely supported probability measures on $W$. Assume that

$$
\inf _{\lambda \in \mathbf{P}(W)} \sup _{V \in \mathfrak{V}} \lambda(V)>0 .
$$

Then, there exists a sequence $\left(V_{j}\right)_{j \geq 1}$ of elements of $\mathfrak{V}$ and a sequence $\left(w_{j}\right)_{j \geq 1}$ of pairwise distinct elements of $W$ such that $\left\{w_{1}, \ldots, w_{n}\right\} \subset V_{n}$ for every $n \geq 1$.

For a proof of Ptak's lemma, see $[\mathrm{Pt}]$ or $[\mathrm{T}]$.

Proof of Theorem 4.9. Let $W:=\left\{-e_{i} ; i \in \mathbb{N}\right\}$ and denote by $\mathfrak{V}$ the family of all subsets $V$ of $W$ such that $x+V \subset Q_{R}^{+}$for some $x \in \bar{B}(0,1+R)$.

Let $\lambda \in \mathbf{P}(W)$, and write $\lambda(w)$ instead of $\lambda(\{w\}), w \in W$. Set

$$
I_{\lambda}:=\left\{i \in \mathbb{N} ; \lambda\left(-e_{i}\right)>0\right\} .
$$

Then $I_{\lambda}$ is a finite set and $\sum_{i \in I_{\lambda}} \lambda\left(-e_{i}\right)=1$.

Since e satisfies the $(\delta, R)$-condition, there exists $J_{\lambda} \subset I_{\lambda}$ and $x \in \bar{B}(0,1+R)$ such that $\sum_{i \in J_{\lambda}} \lambda\left(-e_{i}\right) \geq \delta>0$ and $x+V \subset Q_{R}^{+}$, where $V:=\left\{-e_{i} ; i \in J_{\lambda}\right\}$. Then $V \in \mathfrak{V}$ and $\lambda(J) \geq \delta$.

By Ptak's lemma, there exists an increasing sequence of integers $\left(m_{j}\right)_{n \geq 1}$ and a sequence $\left(V_{j}\right)$ of elements of $\mathfrak{V}$ such that $\left\{-e_{m_{1}}, \ldots,-e_{m_{n}}\right\} \subset V_{n}$ for every $n \geq 1$. So there exists a sequence $\left(x_{n}\right) \subset \bar{B}(0,1+R)$ such that $x_{n}+\left\{-e_{m_{1}}, \ldots,-e_{m_{n}}\right\} \subset Q_{R}^{+}$for all $n \geq 1$. Hence for every $n \geq 1$ and all $j \in\{1, \ldots, n\}$, we have $\left\langle e_{m_{j}}^{*}, x_{n}\right\rangle \geq 1$.

From Theorem 4.9, we easily deduce

Corollary 4.11. If $\mathbf{e}$ is a normalized, 1-unconditional basic sequence satisfying some $(\delta, R)$-condition, then $\mathbf{e}$ has a subsequence which is $(1+R)$-equivalent to the canonical basis of $c_{0}$.

Proof. Let $\left(m_{j}\right)$ and $\left(x_{n}\right)$ be given by Theorem 4.9, and set $\mathbf{f}:=\left(e_{m_{j}}\right)_{j \geq 1}$. By Fact 2.1, we have

$$
\left\|\sum_{j=1}^{n} e_{m_{j}}\right\| \leq\left\|x_{n}\right\| \leq 1+R
$$

for all $n \geq 1$, which implies that $\|\cdot\|^{\mathbf{f}} \leq(1+R)\|\cdot\|_{\infty}^{\mathbf{f}}$. On the other hand, since $\mathbf{f}$ is normalized we also have $\|\cdot\|_{\infty}^{\mathbf{f}} \leq\|\cdot\|^{\mathbf{f}}$, so the subsequence $\mathbf{f}$ is $(1+R)$-equivalent to the canonical basis of $c_{0}$. 
Remark. Of course, the same result holds if $\mathbf{e}$ is only assumed to be semi-normalized, replacing $1+R$ by some constant $M$ depending only on $R$ and on $\inf _{i \geq 1}\left\|e_{i}\right\|$.

If $\mathbf{e}$ is an unconditional basic sequence with a non-Haar null positive cone, then, after the usual renorming to make it 1-unconditional and keeping in mind Proposition 4.1, one can apply Corollary 4.11 to any normalized block-sequence of $\mathbf{e}$. This yields

Corollary 4.12. If $\mathbf{e}$ is an unconditional basic sequence whose positive cone is not Haar null, then every normalized block-sequence $\mathbf{f}$ of $\mathbf{e}$ is $c_{0}$-saturated; that is, any subsequence of $\mathbf{f}$ has a further subsequence equivalent to the canonical basis of $c_{0}$, with uniform bounds on the isomorphism constants.

Another immediate consequence of Corollary 4.11 is the extension of Corollary 4.7 to the case of subsymmetric unconditional basic sequences. Recall that a basic sequence $\mathbf{e}$ is said to be subsymmetric if it is equivalent to all its subsequences. Every symmetric sequence is subsymmetric, but not conversely; see [LiTz].

Corollary 4.13. Up to equivalence, the canonical basis of $c_{0}$ is the only normalized and subsymmetric unconditional basic sequence whose positive cone is not Haar null.

Proof. Let e be such a sequence. Since e is unconditional, Corollary 4.11 implies that e has a subsequence equivalent to the canonical basis of $c_{0}$. Since $\mathbf{e}$ is equivalent to all its subsequences, the result follows.

Finally, we quote the following "decomposition" result for Banach spaces with a nonHaar null positive cone.

Corollary 4.14. Let $\mathbf{e}=\left(e_{i}\right)_{i \geq 1}$ be a normalized unconditional basic sequence, and set $X:=[\mathbf{e}]$. Assume that $Q^{+}(\mathbf{e})$ is not Haar null. Then one can find some constant $M$ and a partition $\left(I_{\lambda}\right)_{\lambda \in \Lambda}$ of $\mathbb{N}$ such that each space $X_{\lambda}:=\left[e_{i} ; i \in I_{\lambda}\right]$ is $M$-isomorphic to $c_{0}$ and $X=\oplus_{\lambda \in \Lambda} X_{\lambda}$, where $\oplus$ denotes an unconditional Schauder decomposition.

Proof. By Corollary 4.12, one can find a constant $C$ such that every subsequence of e has a further subsequence which is $C$-equivalent to the canonical basis of $c_{0}$. By an obvious transfinite induction argument, it follows that one can partition $\mathbb{N}$ as $\mathbb{N}=I_{0} \cup \bigcup_{\lambda \in \Lambda} I_{\lambda}$, where $I_{0}$ is finite (possibly empty) and each space $X_{\lambda}$ is $M$-isomorphic to $c_{0}$. Replacing one set $I_{\lambda}$ by $I_{\lambda} \cup I_{0}$ and using the unconditionality of $\mathbf{e}$, the result follows.

4.4. More on $c_{0}$-saturation. We saw in Corollary 4.12 that unconditional basic sequences with a non-Haar null positive cone have a strong " $c_{0}$-saturation" property. In this section, we elaborate a little bit more on this.

For the sake of brevity, we adopt the following ad hoc terminology: given $M \geq 1$, a normalized basic sequence $\mathbf{e}$ is said to be of type $M c_{0}$ if $\|\cdot\|^{\mathbf{e}} \leq M\|\cdot\|_{\infty}^{\mathbf{e}}$. This, of course, implies that $\mathbf{e}$ is equivalent to the canonical basis of $c_{0}$.

Definition 4.15. Let $\mathbf{e}=\left(e_{i}\right)_{i \geq 1}$ be a normalized basic sequence. We say that 
- e is $M c_{0}$-saturated (for some $M \geq 1$ ) if every subsequence of $\mathbf{e}$ has a further subsequence of type $M c_{0}$.

- e is strongly $M c_{0}$-saturated (for some $M \geq 1$ if the following holds: from every sequence $\left(I_{j}\right)_{j \geq 1}$ of pairwise disjoint subsets of $\mathbb{N}$ for which there exists $N \geq 1$ such that all sequence $\left(e_{i}\right)_{i \in I_{j}}$ are of type $N c_{0}$, it is possible to extract a subsequence $\left(I_{j}^{\prime}\right)_{j \geq 1}$ such that $\left(e_{i}\right)_{i \in \cup_{j \geq 1} I_{j}^{\prime}}$ is of type $N M c_{0}$.

- e is (strongly) $c_{0}$-saturated if it is (strongly) $M c_{0}$-saturated for some constant $M$.

Note that taking each $I_{i}$ to be a singleton in the above definition, we see that strong $M c_{0}$-saturation indeed implies $M c_{0}$-saturation.

Recall also that a space $X$ is said to be $c_{0}$-saturated if every (infinite-dimensional) closed subspace of $X$ admits a further subspace isomorphic to $c_{0}$. Using the BessagaPelczynski selection principle (Lemma 2.3), it is fairly easy to check that if a Banach space $X$ admits a $c_{0}$-saturated (normalized) Schauder basis, then $X$ is $c_{0}$-saturated.

Our aim is to prove the following strengthening of Corollary 4.12.

Theorem 4.16. Let $\mathbf{e}$ be a normalized, 1-unconditional basic sequence. Assume that there exists $\delta, R>0$ such that all normalized block-sequences satisfy the $(\delta, R)$-condition. Then all these block-sequences are strongly $(1+R) c_{0}$-saturated.

From this, we immediately get

Corollary 4.17. If $\mathbf{e}$ is an unconditional basic sequence whose positive cone is not Haar null, then all normalized block-sequences of $\mathbf{e}$ are strongly $c_{0}$-saturated.

The proof of Theorem 4.16 relies on the following lemma.

Lemma 4.18. Let $\mathbf{e}=\left(e_{i}\right)_{i \geq 1}$ be a normalized, 1-unconditional basic sequence, and let $\delta, R>0$. Assume that all normalized block-sequences of e satisfy the $(\delta, R)$-condition. Let also $\mathbf{y}=\left(y_{i}\right)_{i \geq 1}$ be a normalized block-sequence of $\mathbf{e}$. Finally, let $n$ be a positive integer, let $\left(\lambda_{j}\right)_{1 \leq j \leq n}$ be a family of $n$ nonnegative real numbers satisfying $\sum_{j} \lambda_{j}=1$, and let $\left(I_{j}\right)_{1 \leq j \leq n}$ be a family of $n$ pairwise disjoint infinite subsets of $\mathbb{N}$ such that each sequence $\left(y_{i}\right)_{i \in I_{j}}$ is of type $N c_{0}$ for some $N \geq 1$. Then, one can find a set $J \subset\{1, \ldots, n\}$ such that $\sum_{j \in J} \lambda_{j} \geq \delta$ and the sequence $\left(y_{i}\right)_{i \in \cup_{j \in J} I_{j}}$ is of type $N(1+R) c_{0}$.

Proof. For $m \geq 1$ and $1 \leq j \leq n$, let $I_{j}^{m}$ be the set consisting of the first $m$ elements of $I_{j}$, with the convention $I_{j}^{m}:=I_{j}$ if $m \geq\left|I_{j}\right|$. Set $z_{m, j}:=\sum_{i \in I_{j}^{m}} y_{i}\left(\right.$ so that $\left.\left\|z_{m, j}\right\| \leq N\right)$ and $\widehat{z}_{m, j}=\frac{z_{m, j}}{\left\|z_{m, j}\right\|}$.

For any fixed $m$, the sets $I_{j}^{m}$ are pairwise disjoint. Hence $\left(\widehat{z}_{m, j}\right)_{1 \leq j \leq n}$ is a (finite) block-sequence of $\left(y_{i}\right)_{i \geq 1}$ for every $m \geq 1$. So it is also a block-sequence of e, and it is normalized; hence it is 1-unconditional and satisfies the (finite version of) the $(\delta, R)$ condition. Forgetting the dependance on $m$, we denote $\widehat{Q}^{+}$its positive cone in $Z:=$ $\operatorname{span}\left(\widehat{z}_{m, j} ; 1 \leq j \leq n\right)$. 
For $j \in\{1, \ldots, n\}$, denote by $\Delta_{j}$ the Dirac measure at $-\widehat{z}_{m, j}$. Then $\mu=\sum_{j=1}^{n} \lambda_{j} \Delta_{j}$ is a probability measure with finite support contained in $\left\{-\widehat{z}_{m, j} ; 1 \leq j \leq n\right\}$. So there exists $z \in Z$ such that $\mu\left(z+\widehat{Q}_{R}^{+}\right) \geq \delta$, and hence there exists $J^{m} \subset\{1, \ldots, n\}$ such that $\left\langle\widehat{z}_{m, j}^{*}, z\right\rangle \leq-1$ for all $j \in J^{m}$ and $\sum_{j \in J^{m}} \lambda_{j} \geq \delta$. Since $\left(\widehat{z}_{m, j}\right)_{1 \leq j \leq n}$ is 1-unconditional and $\|z\| \leq 1+R$, we deduce from Fact 2.1 that $\left\|\sum_{j \in J^{m}} \widehat{z}_{m, j}\right\| \leq 1+R$; and it follows again from Fact 2.1 that $\left\|\sum_{j \in J^{m}} z_{m, j}\right\| \leq N(1+R)$.

Since there are finitely many sets $J^{m}$, one can find $J \subset\{1, \ldots, n\}$ such that $J=J^{m}$ for infinitely many $m$. Moreover, since the basis constant of $\mathbf{e}$ is equal to 1 , we have, for every $m \geq 1$ and every $m^{\prime} \geq m$,

$$
\left\|\sum_{j \in J} z_{m, j}\right\| \leq\left\|\sum_{j \in J} z_{m^{\prime}, j}\right\| .
$$

It follows that $\left\|\sum_{j \in J} z_{m, j}\right\| \leq N(1+R)$ for every $m \geq 1$. Hence, all finite sums built on $\left(y_{j}\right)_{j \in \cup_{i \in J} I_{i}}$ are bounded in norm by $N(1+R)$, which implies that $\left(y_{j}\right)_{j \in \cup_{i \in J} I_{i}}$ is of type $N(1+R) c_{0}$.

Proof of Theorem 4.16. Since every normalized block-sequence of e satisfies the same assumptions as $\mathbf{e}$, it is enough to show that $\mathbf{e}$ is strongly $(1+R) c_{0}$-saturated.

Let $\left(I_{j}\right)_{j \geq 1}$ be a sequence of pairwise disjoint subsets of $\mathbb{N}$ such that each sequence $\left(e_{i}\right)_{i \in I_{j}}$ is of type $N c_{0}$, for some fixed $N \geq 1$.

Denote by $\mathfrak{V}$ the family of all subsets $V$ of $\mathbb{N}$ such that the basic sequence $\left(e_{i}\right)_{i \in \cup_{j \in V} I_{j}}$ is of type $N(1+R) c_{0}$. Lemma 4.18 ensures that

$$
\inf _{\lambda \in \mathbf{P}(\mathbb{N})} \sup _{V \in \mathfrak{V}} \lambda(V) \geq \delta>0 .
$$

It follows then from Ptak's lemma that there exists a sequence $\left(V_{j}\right)_{i>1}$ of elements of $\mathfrak{V}$, and a sequence $\left(w_{j}\right)_{j \geq 1}$ of pairwise distinct positive integers such that $\left\{w_{1}, \ldots, w_{n}\right\} \subset V_{n}$ for every $n \geq 1$. Then $\left(e_{i}\right)_{i \in \cup_{j \geq 1} I_{w_{j}}}$ is of type $N(1+R) c_{0}$.

4.5. One example. So far, we have shown that for a normalized unconditional basic sequence e, the following implications hold true:

$Q^{+}(\mathbf{e})$ not Haar null $\Longrightarrow$ there exists $\delta, R>0$ such that every normalized blocksequence of $\left(e_{i}\right)_{i \geq 1}$ satisfies the $(\delta, R)$-condition $\Longrightarrow$ every normalized block-sequence of e is strongly $c_{0}$-saturated $\Longrightarrow$ the space $X:=[\mathbf{e}]$ is $c_{0}$-saturated. 
With our "main conjecture" in mind, it is rather tempting to believe that the strong saturation of all block-sequences is already enough to ensure that $\mathbf{e}$ is equivalent to the canonical basis of $c_{0}$. The following result shows that this is not so.

Example 4.19. There exists a normalized, 1-unconditional basic sequence $\mathbf{e}$ which is not isomorphic to the canonical basis of $c_{0}$ but has the property that all its block-sequences are strongly $M c_{0}$-saturated for any $M \geq 1$.

Proof. Not surprisingly, the sequence e will be defined as the "canonical basis" of a $c_{0}$-direct sum of suitably chosen finite-dimensional $\ell_{p}$ spaces.

Let $\left(p_{n}\right)$ be an increasing sequence of real numbers to be chosen later, with $p_{n} \geq 1$ and $\lim _{n \rightarrow \infty} p_{n}=\infty$. We set $X_{n}:=l_{p_{n}}(n)$, and we denote by $\left(e_{i}^{n}\right)_{1 \leq i \leq n}$ the canonical basis of $X_{n}$. Finally, we denote by $X$ the $c_{0}$-direct sum of the spaces $X_{n}$,

$$
X=\oplus_{c_{0}}\left\{X_{n}, n \in \mathbb{N}\right\} .
$$

Denote by $\left(e_{i}\right)_{i \geq 1}$ the "canonical" enumeration of the vectors $e_{i}^{n}$; that is, $e_{1}=e_{1}^{1}$, $e_{2}=e_{1}^{2}, e_{3}=e_{2}^{2}, e_{4}=e_{1}^{3}$ an so on. Then $\left(e_{i}\right)$ is a Schauder basis of $X$. This basis is normalized and 1-unconditional.

For $n \geq 1$, denote by $P_{n}: X \rightarrow X_{n}$ the canonical projection of $X$ onto $X_{n}$. Since $K(\mathbf{e})=1$, we have $\|x\|=\sup \left\{\left\|P_{n}(x)\right\|, n \in \mathbb{N}\right\}$ for every $x \in X$.

We want $\left(e_{i}\right)$ not to be equivalent to the canonical basis of $c_{0}$. For that it is enough to ensure that

and this will hold if the sequence $\left(p_{n}\right)$ satisfies

$$
\limsup _{m \rightarrow \infty}\left\|\sum_{i=1}^{m} e_{i}\right\|=\infty
$$

$$
\lim _{n \rightarrow \infty} n^{1 / p_{n}}=\infty
$$

Having fixed $\left(p_{n}\right)$ in this way, we now show that every normalized block-sequence of $\left(e_{i}\right)_{i \geq 1}$ is strongly $R c_{0}$-saturated for every $R \geq 1$. So let us fix $R \geq 1$, and let $\left(y_{i}\right)_{i \geq 1}$ be a normalized block-sequence of $\left(e_{i}\right)$, and let $\left(I_{i}\right)_{i \geq 1}$ be a sequence of pairwise disjoint subsets of $\mathbb{N}$ such that, for some $N \geq 1$, all sequences $\left(y_{j}\right)_{j \in I_{i}}$ are of type $N c_{0}$.

We claim that for any $k \geq 1$, one can find a positive integer $n_{k}$ such that, for every $n \geq n_{k}$ and every family $\left(x_{i}\right)_{1 \leq i \leq k}$ of disjointly supported elements of $X_{n} \cap \bar{B}(0, N)$, we have

$$
\left\|\sum_{i=1}^{k} x_{i}\right\| \leq N R
$$

Indeed, denoting by $J_{i}$ the support of $x_{i}$ and writing $x_{i}=\sum_{q \in J_{i}} x_{i, q} e_{q}^{n}$, we have

$$
\left\|\sum_{i=1}^{k} x_{i}\right\|=\left[\sum_{i=1}^{k}\left(\sum_{q \in J_{i}}\left|x_{i, q}^{p_{n}}\right|\right)\right]^{1 / p_{n}} \leq\left(\sum_{i=1}^{k} N^{p_{n}}\right)^{1 / p_{n}} \leq N k^{1 / p_{n}} .
$$


So inequality (4.2) is satisfied as soon as $k^{1 / p_{n}} \leq R$, which holds true when $n$ is sufficiently large since $p_{n} \rightarrow \infty$ as $n \rightarrow \infty$.

Since $\left(y_{i}\right)$ is a block-sequence of $\left(e_{i}\right)$, there exists for every $k \geq 1$ an integer $j_{k} \geq 1$ such that, for every $i \geq j_{k}$,

$$
P_{n}\left(y_{i}\right)=0 \quad \text { if } n<n_{k} .
$$

Hence, for every $k \geq 1$, one can find an integer $i_{k} \geq 1$ such that

$$
P_{n}\left(y_{j}\right)=0 \quad \text { for } j \in I_{i_{k}} \quad \text { if } n<n_{k} .
$$

To conclude the proof, it is now enough to show that the sequence $\left(y_{j}\right)_{j \in \cup_{k} \geq 1} I_{i_{k}}$ is of type $N R c_{0}$; and since this sequence is normalized and 1-unconditional, we just have to show that the norm of any finite sum built on it does not exceed $N R$.

Every finite sum $w$ built on $\left(y_{j}\right)_{j \in \cup_{k \geq 1} I_{i_{k}}}$ can be written as

$$
w=\sum_{1 \leq k \leq m} w_{k}
$$

where $m \geq 1$ is an integer and $w_{k}$ is a finite sum built on $\left(y_{j}\right)_{j \in I_{i_{k}}}$. It follows then from the definition of the norm on $X$ that we have

$$
\|w\|=\left\|\sum_{1 \leq k \leq m} w_{k}\right\|=\sup _{n \in \mathbb{N}}\left\|P_{n}\left(\sum_{1 \leq k \leq m} w_{k}\right)\right\| .
$$

For $n \geq n_{m}$ it follows from the definition of $n_{m}$ that $\left\|P_{n}\left(\sum_{1 \leq k \leq m} w_{k}\right)\right\| \leq N R$.

For $n_{i-1} \leq n<n_{i}$, with $1 \leq i \leq m$, we have $P_{n}\left(\sum_{1 \leq k \leq m} w_{k}\right)=P_{n}\left(\sum_{1 \leq k \leq i-1} w_{k}\right)$, and so it follows from the definition of $n_{i-1}$ that $\left\|P_{n}\left(\sum_{1 \leq k \leq m} w_{k}\right)\right\| \leq N R$. Hence, we do get $\|w\| \leq N R$ for any finite sum $w$ built on $\left(y_{j}\right)_{j \in \cup_{k \geq 1} I_{i_{k}}}$, which concludes the proof.

Remark. As it turns out, the above sequence e does not satisfy any $(\delta, R)$-condition, and hence $Q^{+}(\mathbf{e})$ is Haar null. Indeed if $\delta>0$ is given then, for any set $I \subset\{1, \ldots, n\}$ of cardinality $\delta n$ we have $\left\|\sum_{i \in I} e_{i}^{n}\right\| \geq(\delta n)^{1 / p_{n}}$, which tends to $\infty$ by the choice of the sequence $\left(p_{n}\right)$. By Proposition 4.6, it follows that e cannot satisfy any $(\delta, R)$-condition. Therefore, this example leaves our "main conjecture" open.

\section{QUOTIENTS}

We saw in the previous section that if a Banach space $X$ admits an unconditional basis $X$ whose positive cone is not Haar null, then $X$ is $c_{0}$-saturated. One of the main objectives of the present section is to prove the following more precise result.

Theorem 5.1. Let $X$ be a Banach space, and assume that $X$ admits an unconditional basis whose positive cone is not Haar null. Then $X$ has $c_{0}$-saturated quotients; that is, every quotient space of $X$ is $c_{0}$-saturated. 
To put this result into perspective, it is worth mentioning that the class of Banach spaces with $c_{0}$-saturated quotients seems far from being completely understood.

Odell showed in $[\mathrm{O}]$ that the Schreier space $\mathcal{S}$ has this property, and asked whether this holds true for every $c_{0}$-saturated Banach space having an unconditional and shrinking Schauder basis. Leung [Leu] first gave a negative answer to this question, by constructing a $c_{0}$-saturated space with a shrinking unconditional basis admitting a quotient isomorphic to $\ell_{2}$. This was generalized by Gasparis [Ga], who obtained the same result for any $\ell_{p}$, $1<p<\infty$.

Theorem 5.1 just gives one class of examples, possibly consisting of the single space $c_{0}$ (!) In any event, it shows that the $c_{0}$-saturation of quotients is a property strictly weaker than the non-Haar negligibility of the positive cone (by Odell's result and Corollary 4.8), and that the positive cones of the spaces constructed in [Leu] and [Ga] are Haar null.

As a matter of fact, we will deduce Theorem 5.1 from a more general result having little to do with Haar negligibility. Let us first introduce the following terminology.

Definition 5.2. A Banach space $X$ will be said to have property $(\mathrm{P})$ if every weakly null semi-normalized sequence in $X$ admits a subsequence equivalent to the canonical basis of $c_{0}$.

It is clear that in this definition, one can replace "semi-normalized" by "normalized".

Note that if $X$ admits a Schauder basis e then, by Bessaga-Pelczynki's selection principle, property $(\mathrm{P})$ can be tested by looking only at those weakly null normalized sequences which are block-sequence of e. By Theorem 4.16 (or Corollary 4.12) it follows that if the basis $\mathbf{e}$ is unconditional and has a non-Haar null positive cone, then $X$ has property $(\mathrm{P})$.

Note also that if $X$ has separable dual, then every quotient space $E$ of $X$ with property $(\mathrm{P})$ is $c_{0}$-saturated: indeed, any infinite-dimensional subspace of $E$ contains a weakly null normalized sequence (because it has separable dual), and hence a subspace isomorphic to $c_{0}$ by property $(\mathrm{P})$.

From these two remarks, it is clear that the following result implies Theorem 5.1.

Theorem 5.3. Let $X$ be a Banach space admitting an unconditional shrinking Schauder basis. If $X$ has property $(\mathrm{P})$ then all quotients of $X$ have property $(\mathrm{P})$.

Indeed, assume that $X$ is a Banach space admitting an unconditional basis e whose positive cone is not Haar null. Then $X$ has property $(\mathrm{P})$ by Corollary 4.12. Moreover, the basis e is shrinking by corollary 4.2. By Theorem 5.3, all quotients of $X$ have property $(\mathrm{P})$, and hence all these quotients are $c_{0}$-saturated since $X$ has separable dual.

The proof of Theorem 5.3 relies on the following result from [O].

Proposition 5.4. Let $X$ be a Banach admitting a normalized, shrinking and 1-unconditional basis $\left(e_{i}\right)_{i \geq 1}$. Let $T$ be a continuous surjective linear operator from $X$ onto another $B a$ nach space $Y$, and let $C$ be any finite constant such that $\bar{B}_{Y}(0,1) \subseteq C T\left(\bar{B}_{X}(0,1)\right)$. Let also $\left(\varepsilon_{i}\right)_{i \geq 1}$ be a decreasing sequence of positive real numbers tending to 0 . 
Then, given any weakly null sequence $\left(y_{i}\right)_{i \geq 1}$ in the unit ball of $Y$, one can find a subsequence $\left(y_{i}^{\prime}\right)$ of $\left(y_{i}\right)$ and an increasing sequence of integers $\left(p_{i}\right)_{i \geq 0}$ with $p_{0}=0$ satisfying the following property:

For every sequence of real numbers $\left(a_{i}\right)_{i \geq 1}$ such that $\left\|\sum_{i=1}^{\infty} a_{i} y_{i}^{\prime}\right\| \leq 2$, there exists an increasing sequence of integers $\left(r_{i}\right)_{i \geq 0}$ with $r_{0}=0$ and a sequence $\left(x_{i}\right)_{i \geq 1} \subset X$ such that

(1) $p_{i}<r_{i}<p_{i+1}$ for every $i \geq 1$;

(2) $x_{i} \in \operatorname{span}\left(e_{r_{i-1}}, \ldots, e_{r_{i}}\right)$;

(3) $\left\|T\left(x_{i}\right)-a_{i} y_{i}^{\prime}\right\| \leq \varepsilon_{i}$;

(4) the series $\sum x_{i}$ is convergent and $\left\|\sum_{i=1}^{\infty} x_{i}\right\| \leq 2 C$.

Proof of theorem 5.3. Fix a normalized shrinking unconditional basis $\mathbf{e}=\left(e_{i}\right)_{i \geq 1}$ for $X$. Since property $(\mathrm{P})$ is invariant under renormings, we may assume that $\mathbf{e}$ is 1 unconditional.

Let $Y$ be any quotient of $X$, and let $T: X \rightarrow Y$ be the canonical quotient map. Note that $\bar{B}_{Y}(0,1) \subseteq T\left(\bar{B}_{X}(0,1)\right)$.

Let $\left(y_{i}\right)_{i \geq 1}$ be a weakly null normalized sequence in $Y$. Replacing this sequence by a subsequence we may suppose that $\left(y_{i}\right)$ is a basic sequence, with basis constant $K$.

We apply Proposition 5.4 to $\left(y_{i}\right)$, with $\varepsilon_{i}=\frac{1}{2 K} 2^{-i}$; this gives a subsequence $\left(y_{i}^{\prime}\right)$ of $\left(y_{i}\right)$ and an increasing sequence of integers $\left(p_{i}\right)_{i \geq 1}$.

For $i, n \geq 1$ denote by $\delta_{i, n}$ the usual "Kronecker symbol". Then $\left\|\sum_{i=1}^{\infty} \delta_{i, n} y_{i}^{\prime}\right\|=1 \leq 2$ for every $n \geq 1$. Hence one can find for each $n \geq 1$ a sequence $\left(x_{i, n}\right)_{i \geq 1} \subset X$ such that

(1) $x_{i, n} \in\left[e_{r} ; p_{i-1}<r<p_{i+1}\right]$;

(2) $\left\|\sum_{i=1}^{\infty} x_{i, n}\right\| \leq 2$, and in particular $\left\|x_{n, n}\right\| \leq 2$;

(3) $\left\|T\left(x_{n, n}\right)-y_{n}^{\prime}\right\| \leq \varepsilon_{n}$.

Set $u_{i}:=x_{2 i, 2 i}$ and $v_{i}:=y_{2 i}^{\prime}$. Note that by (3), we have $\left\|u_{i}\right\| \geq \frac{1-\varepsilon_{2 i}}{\|T\|} \geq \frac{3}{4\|T\|}$, so that $\left(u_{i}\right)$ is a semi-normalized block-sequence of $\left(e_{i}\right)$.

By the definition of $\left(u_{i}\right)$ and $\left(v_{i}\right)$, we have

$$
\sum_{i=1}^{\infty}\left\|T\left(u_{i}\right)-v_{i}\right\| \leq \sum_{i=1}^{\infty} \varepsilon_{2 i}<\frac{1}{2 K} .
$$

Since $\left(v_{i}\right)_{i \geq 1}$ is a normalized basic sequence, it follows (by the principle of small perturbations, Lemma 2.2) that $\left(T\left(u_{i}\right)\right)$ is a basic sequence equivalent to $\left(v_{i}\right)$ and that for every sequence of real numbers $\left(a_{i}\right)$ such that the series $\sum a_{i} v_{i}$ is convergent we have:

$$
\left(1-2 K \sum_{i=1}^{\infty}\left\|v_{i}-T\left(u_{i}\right)\right\|\right)\left\|\sum_{i=1}^{\infty} a_{i} v_{i}\right\| \leq\left\|\sum_{i=1}^{\infty} a_{i} T\left(u_{i}\right)\right\| \leq 2 K\left\|\sum_{i=1}^{\infty} a_{i} v_{i}\right\| .
$$


In particular, there exists a constant $c>0$ such that for any such sequence $\left(a_{i}\right)$ :

$$
c\left\|\sum_{i=1}^{\infty} a_{i} v_{i}\right\| \leq\|T\| \times\left\|\sum_{i=1}^{\infty} a_{i} u_{i}\right\| .
$$

Now, observe that the sequence $\left(u_{i}\right)$ is weakly null, being a bounded blok-sequence of the shrinking Schauder basis e. Since $\left(u_{i}\right)$ is also semi-normalized and $X$ has property $(\mathrm{P})$, it follows that $\left(u_{i}\right)$ has a subsequence equivalent to the canonical basis of $c_{0}$. The above inequality then implies that the corresponding subsequence of $\left(v_{i}\right)$ is a subsequence of $\left(y_{i}\right)$ equivalent to the canonical basis of $c_{0}$.

To conclude this section (and although this has nothing to do with Haar negligibility), we now show that property $(\mathrm{P})$ is "almost" a three spaces property.

Proposition 5.5. Let $X$ be a Banach space admitting a shrinking unconditional basis, and let $F$ be a closed subspace of $X$. If $F$ et $X / F$ have property $(\mathrm{P})$, then $X$ has property $(\mathrm{P})$.

Proof. Let $\mathbf{e}=\left(e_{i}\right)_{i \geq 1}$ be a normalized, shrinking unconditional basis for $X$, and assume without loss of generality that $\mathbf{e}$ is 1-unconditional. Also, set $Y:=X / F$ and denote by $T: X \rightarrow Y$ the canonical quotient map.

Let $\left(x_{i}\right)_{i \geq 1}$ be a weakly null normalized sequence in $X$. To show that $\left(x_{i}\right)$ admits a subsequence equivalent to the canonical basis of $c_{0}$, we distinguish two cases.

Case 1. $\inf _{i}\left\|T\left(x_{i}\right)\right\|=0$.

In this case, we may assume (upon extracting a subsequence) that $\lim _{i \rightarrow \infty}\left\|T\left(x_{i}\right)\right\|=0$. Then there exists a sequence $\left(f_{i}\right) \subset F$ such that $\left\|x_{i}-f_{i}\right\| \rightarrow 0$. The sequence $\left(f_{i}\right)$ is weakly null, and since $\left(x_{i}\right)$ is normalized we may assume that it is also normalized. Since $F$ has property $(\mathrm{P}),\left(f_{i}\right)$ has a subsequence $\left(f_{i}^{\prime}\right)$ equivalent to the canonical basis of $c_{0}$. By the principle of small perturbations $\left(x_{i}\right)$ has a subsequence equivalent to some subsequence of $\left(f_{i}^{\prime}\right)$, which gives the required result.

Case 2. $\inf _{i}\left\|T\left(x_{i}\right)\right\|>0$.

In this case, $\left(T\left(x_{i}\right)\right)$ is a a weakly null semi-normalized sequence in $Y$. Since $Y$ has property $(\mathrm{P})$, we may assume that this sequence is equivalent to the canonical basis of $c_{0}$. Choose a constant $M>0$ such that for every finite set $I \subset \mathbb{N}$, we have

$$
\left\|\sum_{i \in I} \frac{1}{M} T\left(x_{i}\right)\right\| \leq 2 .
$$

Since $\bar{B}_{Y}(0,1) \subset T\left(\bar{B}_{X}(0,1)\right)$, we may apply Proposition 5.4 with $C:=1$ and $y_{i}:=$ $\frac{1}{M} T\left(x_{i}\right)$, taking e.g. $\varepsilon_{i}:=2^{-i}$. This gives a subsequence $\left(y_{i}^{\prime}\right)_{i \geq 1}$ of $\left(\frac{1}{M} T\left(x_{i}\right)\right)_{i \geq 1}$ and an 
increasing sequence of positive integers $\left(p_{i}\right)_{i \geq 0}$. We denote by $\left(x_{i}^{\prime}\right)_{i \geq 1}$ the subsequence of $\left(x_{i}\right)$ such that

$$
y_{i}^{\prime}=\frac{1}{M} T\left(x_{i}^{\prime}\right)
$$

For each $n \geq 1$, we have $\left\|\sum_{i=1}^{n} y_{i}^{\prime}\right\| \leq 2$. So there exists a sequence $\left(x_{i, n}\right)_{i \geq 1} \subset X$ such that

- $x_{i, n} \in E_{i}:=\left[e_{r} ; p_{2 i-1}<r<p_{2 i+1}\right]$;

- $\left\|T\left(x_{i, n}\right)-y_{i}^{\prime}\right\| \leq \varepsilon_{i}$ if $i \leq n$;

- $\left\|\sum_{i=1}^{\infty} x_{i, n}\right\| \leq 2$, and hence $\left\|\sum_{i \in I} x_{i, n}\right\| \leq 2$ for every finite set $I \subset \mathbb{N}$.

For each fixed $i \geq 1$, the sequence $\left(x_{i, n}\right)_{n \geq 1}$ is a bounded sequence in the finitedimensional space $E_{i}$; in fact $\left\|x_{i, n}\right\| \leq 2$. Hence (by a diagonal argument) we may assume that $\left(x_{i, n}\right)_{n \geq 1}$ converges to some $\bar{x}_{i} \in E_{i}$ for every $i \geq 1$. Then $\left\|\bar{x}_{i}\right\| \leq 2$ and $\left\|T\left(\bar{x}_{i}\right)-y_{i}^{\prime}\right\| \leq \varepsilon_{i}$. Since $\left(y_{i}^{\prime}\right)$ is semi-normalized, the latter inequality implies that $\lim \inf _{i}\left\|\bar{x}_{i}\right\|>0$; hence, we may assume that $\left(\bar{x}_{i}\right)$ is semi-normalized.

Now set $u_{i}:=\bar{x}_{2 i}$ and $v_{i}:=\frac{1}{M} x_{2 i}^{\prime}$. Observe that $\left(u_{i}\right)$ is a semi-normalized blocksequence of e. For every finite set $I \subset \mathbb{N}$, we have

$$
\left\|\sum_{i \in I} u_{i}\right\|=\lim _{n \rightarrow \infty}\left\|\sum_{i \in I} x_{2 i, n}\right\| \leq 2 .
$$

Since $\left(u_{i}\right)$ is unconditional (being a block-sequence of $\mathbf{e}$ ) and semi-nomalized, it follows that $\left(u_{i}\right)$ is equivalent to the canonical basis of $c_{0}$. Moreover, since $\left\|T\left(\bar{x}_{i}\right)-y_{i}^{\prime}\right\| \leq \varepsilon_{i}$ for all $i$, we have

$$
\lim _{i \rightarrow \infty} T\left(u_{i}-v_{i}\right)=0 .
$$

Set $z_{i}:=u_{i}-v_{i}$. If there exists a subsequence of $\left(z_{i}\right)_{i>1}$ which converges to 0 , the principle of small perturbations tells us that some subsequence of $\left(v_{i}\right)$ is equivalent to $\left(u_{i}\right)$, i.e to the canonical basis of $c_{0}$, and the conclusion follows since $v_{i}=\frac{1}{M} x_{i}$. Otherwise (disgarding finitely many terms) the sequence $\left(z_{i}\right)$ is semi-normalized, and hence we may apply Case 1 to it; so there exists a subsequence of $\left(z_{i}\right)$ which is equivalent to the canonical basis of $c_{0}$. It is then easily checked that the same property holds true for $\left(v_{i}\right)$, and hence for $\left(x_{i}\right)$.

\section{Positive cones and Gaussian measures}

6.1. Gaussian test measures. In this section we intend to produce "explicit" test measures for Haar null positive cones. More precisely we will see that under reasonable assumptions, it is possible to construct test measures which are Gaussian measures.

Gaussian measures on Banach spaces may be defined as follows: a Borel probability measure $\mu$ on a separable Banach space $X$ is Gaussian if and only if it is the distribution 
of an $X$-valued random variable of the form

$$
\xi(\omega)=x_{0}+\sum_{i=1}^{\infty} g_{i}(\omega) x_{i},
$$

where $x_{i} \in X$, the $g_{i}$ are independent real-valued random variable (defined on the same probability space $(\Omega, \mathfrak{A}, \mathbb{P}))$ having standard Gaussian distribution $\gamma=\frac{1}{\sqrt{2 \pi}} e^{-\frac{t^{2}}{2}} d t$, and the series is almost surely convergent. This means that for any Borel set $A \subset X$, we have

$$
\mu(A)=\mathbb{P}(\xi \in A) .
$$

A Gaussian measure $\mu$ is said to be centred if $x_{0}=0$ in the above representation, which means that $\int_{X} x^{*} d \mu=0$ for every continuous linear functional $x^{*} \in X^{*}$. The measure $\mu$ is nondegenerate if $\mu(V)>0$ for every open set $V \neq \emptyset$; in the above representation, this means that the linear span of the $x_{i}, i \geq 1$ is dense in $X$. For more information on Gaussian measures, we refer to [Bog] or [CTV].

In the remaining of this section, we fix a normalized basic sequence e whose positive cone is Haar null, and we denote by $X$ the Banach space generated by e. For the sake of notational simplicity, we write $Q^{+}$instead of $Q^{+}(\mathbf{e})$. For technical reasons, we will in fact mostly deal with the negative cone $Q^{-}:=-Q^{+}$.

We will consider Gaussian measures of a very special type. Let us fix once and for all a sequence independent real-valued random variable (defined on the same probability space $(\Omega, \mathfrak{A}, \mathbb{P}))$ having standard Gaussian distribution. We denote by $\mathfrak{R}(\mathbf{e})$ the family of all sequences of positive real numbers $\mathfrak{r}=\left(r_{i}\right)_{i \geq 1}$ such that the random series $\sum r_{i} g_{i} e_{i}$ is almost surely convergent. If $\mathfrak{r} \in \mathfrak{R}(\mathbf{e})$, the corresponding Gaussian measure is denoted by $\mu_{\mathrm{r}}$ :

$$
\mu_{\mathfrak{r}} \sim \sum r_{i} g_{i} e_{i}
$$

Any such measure will be called a diagonal Gaussian measure. Note that $\mu_{\mathfrak{r}}$ is centred, and also nondegenerate since $r_{i} \neq 0$ for all $i$.

We restrict ourselves to diagonal measure because this allows to compute very easily the measure of a translate of the negative cone. Indeed, if $x=\sum_{1}^{\infty} x_{i} e_{i} \in X$ then, by independance, we have

$$
\mu\left(x+Q^{-}\right)=\prod_{i \geq 1} \mathbb{P}\left(g_{i} \leq \frac{x_{i}}{r_{i}}\right) .
$$

Having set these notations, we are going to prove the following result.

Theorem 6.1. Assume that the basic sequence $\mathbf{e}$ is unconditional. Let $\mathfrak{r} \in \mathfrak{R}(\mathbf{e})$, and assume that there exists a permutation $\pi_{0}$ of the integers and a constant $\alpha>0$ such that $r_{\pi_{0}(i)}=O\left(i^{-\alpha}\right)$. Then $\mu_{\mathfrak{r}}$ is a test measure for $Q^{-}=Q^{-}(\mathbf{e})$ if and only if the series $\sum \log (i)^{1 / 2} r_{\pi_{0}(i)} e_{\pi_{0}(i)}$ is divergent. 
When $\mathbf{e}$ is the canonical basis of $\ell_{p}$, it is well-known that a Gaussian series $\sum r_{i} g_{i} e_{i}$ is almost surely convergent in $\ell_{p}$ if and only if $\sum_{1}^{\infty}\left|r_{i}\right|^{p}<\infty$; see [CTV, Chapter V]. Applying Theorem 6.1, we get

Corollary 6.2. Assume that $\mathbf{e}$ is the canonical basis of $\ell_{p}$, and let $\mathfrak{r}=\left(r_{i}\right)$ be a sequence of positive real numbers such that $\sum_{1}^{\infty} r_{i}^{p}<\infty$. Denote by $\left(\bar{r}_{i}\right)$ the decreasing rearrangement of $\left(r_{i}\right)$. Then, the measure $\mu_{\mathfrak{r}}$ is a test measure for $Q^{-}$if and only if

$$
\sum_{i \geq 1} \log (i)^{p / 2} \bar{r}_{i}^{p}=\infty
$$

Proof. This follows immediately from Theorem 6.1 because $\bar{r}_{i}=o\left(i^{-1 / p}\right)$.

To prove Theorem 6.1, we basically have to determine whether an expression like (6.1) above can be positive or not. The following remark shows that it is enough to consider vectors $x \in X$ having positive coefficients on the basis e. The set of all such vectors $x$ will be denoted by $Q^{++}$:

$$
Q^{++}=\left\{x=\sum_{i=1}^{\infty} x_{i} e_{i} \in X ; x_{i}>0 \text { for all } i \geq 1\right\} .
$$

Remark 6.3. Let $\mathfrak{r} \in \mathfrak{R}(\mathbf{e})$. For any $a \in X$, one can find $x \in Q^{++}$such that $\mu_{\mathfrak{r}}\left(x+Q^{-}\right) \geq$ $\mu_{\mathfrak{r}}\left(a+Q^{-}\right)$. In particular, if $\mu_{\mathfrak{r}}$ is not a test measure for $Q^{-}$then there exists $x \in Q^{++}$ such that $\mu_{\mathfrak{r}}\left(x+Q^{-}\right)>0$.

Proof. Write $a=\sum_{1}^{\infty} a_{i} e_{i}$ and assume without loss of generality that $\mu_{\mathfrak{r}}\left(a+Q^{-}\right)>0$. Set $I_{a}^{-}=\left\{i \in \mathbb{N} ;, a_{i} \leq 0\right\}$ and $I_{a}^{+}=\left\{i \in \mathbb{N} ; a_{i}>0\right\}$; then

$$
\mu_{\mathfrak{r}}\left(a+Q^{-}\right)=\prod_{i \in I_{a}^{-}} \mathbb{P}\left(g_{i} \leq \frac{a_{i}}{r_{i}}\right) \times \prod_{i \in I_{a}^{+}} \mathbb{P}\left(g_{i} \leq \frac{a_{i}}{r_{i}}\right),
$$

and hence

$$
\mu_{\mathfrak{r}}\left(a+Q^{-}\right) \leq \prod_{i \in I_{a}^{-}} \mathbb{P}\left(g_{i} \leq \frac{a_{i}}{r_{i}}\right) \leq 2^{-\left|I_{a}^{-}\right|} .
$$

Hence, the set $I_{a}^{-}$is finite. So if we set $x_{i}:=1$ if $i \in I_{a}^{-}$and $x_{i}:=a_{i}$ if $i \in I_{a}^{+}$, then $x:=\sum_{i \geq 1} x_{i} e_{i}$ is a well-defined element of $Q^{++}$such that $\mu_{\mathfrak{r}}\left(x+Q^{-}\right) \geq \mu_{\mathfrak{r}}\left(a+Q^{-}\right)$.

In view of this remark, in what follows we will only consider translates of the negative cone $Q^{-}$by elements of $Q^{++}$.

Lemma 6.4. Let $\mathfrak{r}=\left(r_{i}\right) \in \mathfrak{R}(\mathbf{e})$, and let $x=\sum_{i \geq 1} x_{i} e_{i} \in Q^{++}$. Then

$$
\mu_{\mathfrak{r}}\left(x+Q^{-}\right)>0 \quad \text { if and only if } \sum_{i \geq 1} \frac{e^{-\frac{1}{2}\left(x_{i} / r_{i}\right)^{2}}}{\left(x_{i} / r_{i}\right)}<\infty \text {. }
$$


Proof. We have $\mu\left(x+Q^{-}\right)=\prod_{i \geq 1} \mathbb{P}\left(g_{i} \leq \frac{x_{i}}{r_{i}}\right)=\prod_{i \geq 1}\left(1-\mathbb{P}\left(g_{i} \geq \frac{x_{i}}{r_{i}}\right)\right)$. Therefore, $\mu\left(x+Q^{-}\right)>0$ if and only if

$$
\sum_{i \geq 1} \mathbb{P}\left(g_{i} \geq \frac{x_{i}}{r_{i}}\right)<\infty
$$

Set $g(t):=\frac{e^{-t^{2} / 2}}{\sqrt{2 \pi}}$ and $G(a)=\int_{a}^{\infty} g(t) d t, a>0$. Then $\mathbb{P}\left(g_{i} \geq \frac{x_{i}}{r_{i}}\right)=G\left(\frac{x_{i}}{r_{i}}\right)$. Now, it is well-known that

and the lemma follows.

$$
G(a) \sim \frac{e^{-a^{2} / 2}}{\sqrt{2 \pi} a} \quad \text { as } a \rightarrow \infty
$$

This lemma allows now to state the following "comparison criterion".

Lemma 6.5. Let $\mathfrak{r} \in \mathfrak{R}(\mathbf{e})$, and let $x \in Q^{++}$. Let also $\pi$ be a permutation of the integers.

- If $\limsup _{i \rightarrow \infty}\left(\frac{x_{\pi(i)}}{r_{\pi(i)}} \log (i)^{-1 / 2}\right)<\sqrt{2}$, then $\mu_{\mathfrak{r}}\left(x+Q^{-}\right)=0$.

- If $\liminf _{i \rightarrow \infty}\left(\frac{x_{\pi(i)}}{r_{\pi(i)}} \log (i)^{-1 / 2}\right)>\sqrt{2}$, then $\mu_{\mathfrak{r}}\left(x+Q^{-}\right)>0$.

Proof. Set $b_{i}:=\frac{x_{i}}{r_{i}}$ and $a_{i}=\frac{x_{\pi(i)}}{r_{\pi(i)}}$. Then $\mu\left(x+Q^{-}\right)>0$ if and only if $\sum_{1}^{\infty} \frac{e^{\left(b_{i}\right)^{2} / 2}}{b_{i}}<\infty$, which is equivalent to the convergence of the series $\sum \frac{e^{-\left(a_{i}\right)^{2} / 2}}{a_{i}}$. We need the following

Fact. For any $M>0$, set $h_{M}(t):=g\left(M \log (t)^{1 / 2}\right)$, where $g(t)=\frac{e^{-t^{2} / 2}}{t}$. Then the series $\sum_{i \geq 1} h_{M}(i)$ is convergent if $M>\sqrt{2}$, and divergent if $M<\sqrt{2}$

Proof of Fact. The fonction $h_{M}$ is positive and decreasing on $(0, \infty)$, and so the convergence of the series $\sum_{i \geq 1} h_{M}(i)$ is equivalent to the convergence of the integral $\int_{1}^{\infty} h_{M}(t) d t$. A simple computation gives $h_{M}^{\prime}(t)=-h_{M}(t)\left(\frac{M^{2}}{2 t}+\frac{1}{2 t \log (t)}\right)$, and so $\frac{h_{M}^{\prime}(t)}{h_{M}(t)} \sim-\frac{M^{2}}{2 t}$ as $t \rightarrow \infty$. It follows that the integral $\int_{1}^{\infty} h_{M}(t)$ is convergent if $M>\sqrt{2}$ and divergent if $M<\sqrt{2}$.

If $\liminf _{i \rightarrow \infty}\left(a_{i} \log (i)^{-1 / 2}\right)>\sqrt{2}$, take $M>\sqrt{2}$ such that $a_{i} \geq M \log (i)^{1 / 2}$ for $i$ sufficiently large, say $i \geq i_{M}$. Since $g(t)=\frac{e^{-t^{2} / 2}}{t}$ is decreasing on $(0, \infty)$, we obtain

$$
\sum_{i \geq i_{M}} \frac{e^{-\left(a_{i}\right)^{2} / 2}}{a_{i}}=\sum_{i \geq i_{M}} g\left(a_{i}\right) \leq \sum_{i \geq i_{M}} g\left(M \log (i)^{1 / 2}\right)=\sum_{i \geq i_{M}} h_{M}(i),
$$

and hence $\mu_{\mathfrak{r}}\left(x+Q^{-}\right)>0$ by the above Fact.

If $\limsup \left(a_{i} \log (i)^{-1 / 2}\right)>\sqrt{2}$, one gets in the same way that $\mu_{\mathfrak{r}}\left(x+Q^{-}\right)>0$. 
From Lemma 6.5, we immediately deduce

Corollary 6.6. Let $\mathfrak{r} \in \mathfrak{R}(\mathbf{e})$. If there exists a permutation $\pi$ of the integers such that the series $\sum_{i \geq 1} \log (i)^{1 / 2} r_{\pi(i)} e_{\pi(i)}$ converges in $X$, then $\mu_{\mathfrak{r}}$ is not a test measure for $Q^{-}$.

Proof. If we set $x_{M}:=M \sum_{i \geq 1} \log (i)^{1 / 2} r_{\pi(i)} e_{\pi(i)}$, then $\mu_{\mathfrak{r}}\left(x_{M}+Q^{-}\right)>0$ for any $M>$ $\sqrt{2}$.

The next lemma gives a kind of converse to Corollary 6.6.

Lemma 6.7. Assume that the basic sequence $\mathbf{e}$ is unconditional, and let $\mathfrak{r} \in \mathfrak{R}(\mathbf{e})$. If $\mu_{\mathfrak{r}}$ is not a test measure for $Q^{-}$then, for every permutation $\pi$ of the integers and every $\alpha>0$, there exists a set $I=I_{\pi, \alpha} \subset \mathbb{N}$ such that $\sum_{i \in I} \frac{1}{i^{\alpha} \log (i)^{1 / 2}}<\infty$ and the series $\sum_{i \notin I} \log (i)^{1 / 2} r_{\pi(i)} e_{\pi(i)}$ is convergent.

Proof. Assume that $\mu$ is not a test measure $Q^{-}$, and choose a point $x \in Q^{++}$such that $\mu\left(x+Q^{-}\right)>0$. Write $x=\sum_{i \geq 1} a_{i} r_{i} e_{i}$, so that $a_{i}>0$ for all $i$. By Lemma 6.4, we know that the series $\sum \frac{e^{-\left(a_{i}\right)^{2} / 2}}{a_{i}}$ is convergent.

Now, fix $\alpha>0$ and a permutation $\pi$ of the integers. Set

$$
I:=\left\{i \in \mathbb{N} ; a_{\pi(i)} \leq \sqrt{2 \alpha} \log (i)^{1 / 2}\right\} .
$$

By unconditionality and the definition of $I$, the series $\sum_{i \notin I} \log (i)^{1 / 2} r_{\pi(i)} e_{\pi(i)}$ is convergent. Moreover, we have

$$
\sum_{i \in I} \frac{1}{i^{\alpha} \log (i)^{1 / 2}}=\sum_{i \in I} \frac{e^{-\alpha \log (i)}}{\log (i)^{1 / 2}} \leq \sum_{i \in I} \frac{e^{-\left(a_{\pi(i)}\right)^{2} / 2}}{a_{\pi(i)}}<\infty .
$$

We can now give the

Proof of Theorem 6.1. Taking $\alpha$ smaller if necessary, we may assume that in fact

$$
r_{\pi_{0}(i)}=O\left(\frac{1}{i^{\alpha} \log (i)}\right) .
$$

By Corollary 6.6, we know that if $\mu_{\mathfrak{r}}$ is a test measure for $Q^{-}$, then $\sum \log (i)^{1 / 2} r_{\pi_{0}(i)} e_{\pi_{0}(i)}$ is divergent.

Conversely, assume that $\mu_{\mathfrak{r}}$ is not a test measure for $Q^{-}$. Then, by Lemma 6.7 , there exists a set $I \subset \mathbb{N}$ such that $\sum_{i \in I} \frac{1}{i^{\alpha} \log (i)^{1 / 2}}<\infty$ and the series $\sum_{i \notin I} \log (i)^{1 / 2} r_{\pi_{0}(i)} e_{\pi_{0}(i)}$ is convergent.

Since $r_{\pi_{0}(i)}=O\left(\frac{1}{i^{\alpha} \log (i)}\right)$, we have $\sum_{i \in I}\left\|\log (i)^{1 / 2} r_{\pi_{0}(i)} e_{\pi_{0}(i)}\right\|=\sum_{i \in I} \log (i)^{1 / 2} r_{\pi_{0}(i)}<$ $\infty$. So the series $\sum_{i \in I} \log (i)^{1 / 2} r_{\pi_{0}(i)} e_{\pi_{0}(i)}$ is convergent, and altogether the whole series $\sum \log (i)^{1 / 2} r_{\pi_{0}(i)} e_{\pi_{0}(i)}$ is convergent. 
6.2. Positive cones are not Gauss null. Besides Haar negligibility, there are many natural notions of smallness for subsets of Banach spaces. One of the most useful ones is Gauss negligibility, which was introduced by $\mathrm{R}$. R. Phelps in [Ph]. A Borel set $A$ in a separable Banach space $X$ is said to be Gauss null if $\mu(A)=0$ for every nondegenerate Gaussian measure $\mu$ on $X$.

Since the family of all Gaussian measures on $X$ is translation-invariant, any Gauss null set is obviously Haar null; more precisely, a Borel set $A \subset X$ is Gauss null exactly when every nondegenerate Gaussian measure on $X$ is a test measure for $A$.

Gauss negligibility is in fact a much stronger notion of smallness than Haar negligibility. For example, a compact set needs not be Gauss null; see [BeLi]. More in the spirit of the present paper, we have the following result.

Proposition 6.8. Positive cones determined by basic sequences are never Gauss null.

Proof. Let $\mathbf{f}=\left(f_{i}\right)_{i \geq 1}$ be any normalized basic sequence, and set $X:=[\mathbf{f}]$.

Let also $\left(e_{i}\right)_{i \geq 1}$ be the canonical basis of $\ell_{2}$, and fix any vector $x=\sum_{i \geq 1} x_{i} e_{i} \in \ell_{2}$ such that $x_{i} \neq 0$ for all $i \geq 1$. Let $\left(\alpha_{i}\right)$ be a sequence of positive numbers such that the formula

$$
T\left(\sum_{i \geq 1} y_{i} e_{i}\right):=\sum_{i \geq 1} \alpha_{i} \operatorname{sgn}\left(x_{i}\right) y_{i} f_{i}
$$

defines a bounded operator $T: \ell_{2} \rightarrow X$. (For example we can take any square-summable sequence $\left.\left(\alpha_{i}\right)\right)$. Note that the operator $T$ has dense range since $\alpha_{i} x_{i} \neq 0$ for all $i$.

Now, choose a sequence $\left(a_{i}\right)_{i \geq 1} \subset(0,1)$ such that $\prod_{i \geq 1}\left(1-a_{i}\right)>0$, and a sequence of real numbers $\left(r_{i}\right)$ such that $0<\frac{r_{i}}{x_{i}} \leq \sqrt{a_{i}}$ for all $i \geq 1$. The series $\sum_{i \geq 1} r_{i} e_{i}$ is convergent in $\ell_{2}$ and so the Gaussian series $\sum_{i \geq 1} r_{i} g_{i} e_{i}$ is almost surely convergent. Let $\nu$ be the associated Gaussian measure on $\ell_{2}$ :

$$
\nu \sim \sum_{i=1}^{\infty} r_{i} g_{i} e_{i}
$$

Since the operator $T: \ell_{2} \rightarrow X$ has dense range, the measure

$$
\mu:=\nu \circ T^{-1} \sim \sum_{i=1}^{\infty} r_{i} g_{i} T e_{i}
$$

is a nondegenerate Gaussian measure on $X$.

Set

$$
H:=\left\{y=\sum_{i \geq 1} y_{i} e_{i} \in \ell_{2} ; \frac{y_{i}}{r_{i}} \geq 0 \text { for all } i\right\} .
$$


By the independence of the random variables $g_{i}$, we have

$$
\begin{aligned}
\nu(-x+H) & =\prod_{i \geq 1} \mathbb{P}\left(g_{i} \geq-x_{i} / r_{i}\right) \\
& \geq \prod_{i \geq 1} \mathbb{P}\left(\left|g_{i}\right| \leq x_{i} / r_{i}\right) \\
& \geq \prod_{i \geq 1}\left(1-\mathbb{P}\left(\left|g_{i}\right|>x_{i} / r_{i}\right)\right) .
\end{aligned}
$$

Since $\mathbb{P}\left(\left|g_{i}\right|>x_{i} / r_{i}\right) \leq r_{i}^{2} / x_{i}^{2}$ by Chebishev's inequality, it follows that

$$
\nu(-x+H) \geq \prod_{i \geq 1}\left(1-\frac{r_{i}^{2}}{x_{i}^{2}}\right) \geq \prod_{i \geq 1}\left(1-a_{i}\right)>0 .
$$

On the other hand, since $T(H) \subset Q^{+}(\mathbf{f})$ by the definition of $T$ and since $T$ is one-toone, we have

$$
\mu\left(T(-x)+Q^{+}(\mathbf{f})\right) \geq \mu(T(-x+H))=\nu(-x+H) .
$$

So $\mu\left(T(-x)+Q^{+}(\mathbf{f})\right)>0$, and hence $Q^{+}(\mathbf{f})$ is not Gauss null.

Remark. Another way of proving Proposition 6.8 is by using a deep result of M. Csörnyei, according to which Gauss negligibility is equivalent to cube negligibility (see [Cs] or $[\mathrm{BeLi}])$. Indeed, it is not difficult to check directly that the positive cone of a Schauder basis is never cube-null; see [Mo, Prop 3.5.2]

\section{Some QUESTIONS}

(1) Let us say that a Borel set $A$ in a Polish abelian group $G$ compactivorous if for every compact set $K \subset G$, one can find $x \in G$ and an open set $V$ such that $V \cap K \neq \emptyset$ and $x+(V \cap K) \subset A$. Does there exist a non-Haar null positive cone (in some Banach space) which is not compactivorous?

(1') Does there exist any closed convex non-Haar null set which is not compactivorous? Note that for a quite large class of Polish abelian groups $G$, one can find a $G_{\delta}$ set in $G$ which is non-Haar null and non-compactivorous (see [M1, Proposition 1.2.2]), but it does not seem clear that there always exists a closed set with these properties.

(2) What can be said of a Banach lattice whose positive cone is not Haar null?

(3) Let e a normalized unconditional basic sequence. Assume that there exists $\delta, R>$ 0 such that all block-sequences of e satisfy the $(\delta, R)$-condition. Does this imply that $\mathbf{e}$ has to be equivalent to the canonical basis of $c_{0}$ ?

(3') In the situation of (3), is it at least true that $Q^{+}(\mathbf{e})$ is not Haar null?

(4) Regarding our "main conjecture", one may ask if the following weaker result holds true: if $\mathbf{e}$ is an unconditional basic sequence whose positive cone is not Haar null, then the Banach space [e] is isomorphic to a subspace of $c_{0}$. The methods of [GKL] might be relevant here. 
(5) Is property $(\mathrm{P})$ a three spaces property?

(6) Let $\mathbf{e}$ be basic sequence with a Haar null positive cone. Assume that $Q^{+}(\mathbf{e})$ admits a Gaussian test measure. Does it admit a diagonal such measure?

(7) Does there exist a Haar null positive cone which does not admit any Gaussian test measure?

(8) It is known that if $G$ is a non-locally compact Polish abelian group, then the family of all closed Haar null sets of $G$ is extremely complicated from a descriptive point of view; see [So] and [SR]. What is the descriptive complexity of the family of all basic sequences having a Haar null positive cone?

\section{REFERENCES}

[AK] F. Albiac and N. J. Kalton, Topics in Banach space theory. Springer GTM 233 (2006).

[BeLi] Y. Benyamini, and J. Lindenstrauss, Geometric Nonlinear Functional Analysis. AMS Colloquium Publications 48 (2000).

[Bog] V. I. Bogachev : Gaussian mesures. AMS Mathematical Surveys and Monographs 62 (1998).

[BorN] J. Borwein and D. Noll, Second order differentiability of convex functions in Banach spaces. Trans. Amer. Math. Soc 342 (1994), 43-81.

[BouTz] J. Bourgain and L. Tzafriri, On a problem of Kadison and Singer. J. Reine. Angew. Math. 420 (1991), 1-43.

[CTV] S. A. Chobanyan, V. I.Tarieladze and N. N. Vakhania, Probability distributions on Banach spaces. Kluwer (1987).

[Chr1] J. P. R Christensen, On sets of Haar measure zero in abelian Polish groups. Israel J. Math. 13 (1972), 255-260.

[Chr2] J. P. R Christensen, Topology and Borel Structures. North-Holland (1974).

[Cs] M. Csörnyei, Aronszajn null and Gaussian null sets coincide. Israel J. Math. 111 (1999),191-201.

[Ga] I. Gasparis, New examples of $c_{0}$-saturated Banach spaces. J. Funct. Anal. 256 (2009), 3830-3840.

[GKL] G. Godefroy, N. J. Kalton and G. Lancien, Subspaces of $c_{0}(\mathbb{N})$ and Lipschitz isomorphisms. Geom. Funct. Anal. 10 (2000), 798-820.

[HSY] B. R. Hunt, T. Sauer and J. A. Yorke : Prevalence: a translation invariant "almost every" on infinite-dimensional spaces, Bull. Amer. Math. Soc. 27 (1992), 217-238.

[LMP] P. Lefèvre, É. Matheron and A. Primot, Smoothness, asymptotic smoothness and the BlumHanson property. Israel J. Math., to appear.

[Leu] D. H. Leung, On $c_{0}$-saturated Banach spaces. Illinois J. Math. 39 (1995), 15-29.

[LiTz] J. Lindenstrauss and L. Tzafriri, Classical Banach Spaces I. Springer (1977).

[M1] E. Matouskova, Convexity and Haar null sets. Proc. Amer. Math. Soc. 124 (1997), 1083-1090.

[M2] E. Matouskova, The Banach-Saks property and Haar null sets. Comment. Math. Univ. Carolin. 39 (1998), 71-80.

[M3] E. Matouskova : Translating finite sets into convex sets. Bull. London Math. Soc. 33 (2001), $711-714$.

[MS] E. Matouskova and C. Stegall, A characterization of reflexive Banach spaces. Proc. Amer. Math. Soc. 124 (1996), 1083-1090.

[Mo] P. Moreau, Notions de petitesse, géométrie des espaces de Banach et hypercyclicité. Thèse, Université de Bordeaux (2009).

[O] E. Odell, On quotients of Banach spaces having shrinking unconditional bases. Illinois J. Math. 36 (1992), 681-695.

[Ph] R. R. Phelps, Gaussian null sets and differentiability of Lipschitz maps on Banach spaces. Pacific J. Math. 77 (1978), 523-531. 
[Pt] V. Ptak, A combinatorial lemma on the existence of convex means and its application to weak compactness. Proc. Symp. Pure Math. VII (1963), 437-450.

[SR] J.Saint-Raymond : Quasi-bounded trees and analytic inductions, Fund. Math. 191 (2006), 175185.

[So] S. Solecki : Haar null and non-dominating sets. Fund. Math. 170 (2001), 197-217.

[T] S.Todorcevic, Topics in topology. Springer LNM 1652 (1997).

IMB, UMR 5251, Université de Bordeaux, 351, Cours de la Libération, 33405 Talence (FRANCE).

E-mail address: esterle@math.u-bordeaux1.fr

Laboratoire de Mathématiques de Lens, Université D’Artois, Rue Jean Souvraz S. P. 18, 62307 LENS (FRANCE).

E-mail address: etienne.matheron@euler.univ-artois.fr

Lycée La Pérouse-Kerichen, Rue Prince de Joinville - BP 82517, 29225 Brest Cedex 2 E-mail address: Pierre.Moreau@ac.rennes.fr 